ISSN-L 1561-0837; elSSN: 1727-9933

Universidad Nacional Mayor de San Marcos

\title{
A new species of Thomasomys Coues, 1884 (Rodentia: Sigmodontinae) from the montane forests of northern Peru with comments on the "aureus" group
}

\author{
Una especie nueva de Thomasomys Coues, 1884 (Rodentia: Sigmodontinae) de los bos- \\ ques montanos del norte de Perú con comentarios sobre el grupo "aureus"
}

\author{
Dennisse Ruelas*1,2,3 \\ http://orcid.org/0000-0002-3793-8639 \\ druelasp@unmsm.edu.pe \\ Víctor Pacheco ${ }^{1,2}$ \\ http://orcid.org/0000-0002-1005-135X \\ vpachecot@unmsm.edu.pe

\section{*Corresponding author}

1 Museo de Historia Natural, Universidad Nacional Mayor de San Marcos, Av. Arenales 1256, Jesús María, Lima 15072, Perú.

2 Instituto de Ciencias Biológicas "Antonio Raimondi", Facultad de Ciencias Biológicas, Universidad Nacional Mayor de San Marcos, Av. Venezuela, Cdra. 34 S/N, Ciudad Universitaria, Lima 01, Lima, Perú

3 Institut des Sciences de l'Evolution (ISEM, UMR 5554 CNRS-IRD-UM), Université de Montpellier, Place E. Bataillon CC 064 -34095, Montpellier Cedex 5, France.

\section{Citación}

Ruelas D, Pacheco V. 2021. A new species of Thomasomys Coues, 1884 (Rodentia: Sigmodontinae) from the montane forests of northern Peru with comments on the "aureus" group. Revista peruana de biología 28(3): e19912 (Agosto 2021). doi: http://dx.doi. org/10.15381/rpb.v28i3.19912

\section{Presentado: $\quad 22 / 03 / 2021$ \\ Aceptado: 22/06/2021 \\ Publicado online: 30/08/2021}

Editor:

Leonardo Romero

\begin{abstract}
We describe a new species of the cricetid rodent Thomasomys (Sigmodontinae) of the "aureus" group based on four specimens collected from Carmen de la Frontera, Piura Department, Peru. This new species has a very long and white tail, very long mystacial vibrissae that extend posteriorly beyond the pinnae, and a distinctive cranial and dental morphology that differ from any other known species of Thomasomys. It is also one of the largest species of Thomasomys, exceeded in size only by T. apeco. A phylogenetic analysis using sequences of $C y t b$ recovered this species within a non-monophyletic "aureus" group with a genetic distance between $5.47 \%$ (with T. auricularis) to $10.17 \%$ (with Thomasomys sp. 1). In addition to this finding, the phylogenetic position of T. apeco, T. praetor, and T. pyrrhonotus are presented for the first time, prompting a discussion on the nature of the "aureus" group.
\end{abstract}

\section{Resumen}

Describimos una nueva especie de roedor cricétido Thomasomys (Sigmodontinae) del grupo "aureus" a partir de cuatro especímenes colectados en Carmen de la Frontera, en el departamento de Piura, Perú. Esta nueva especie tiene una cola muy larga y blanca, vibrisas mistaciales muy largas que sobrepasan ampliamente el pabellón auricular y una morfología craneal y dental distintiva que difiere de cualquier otra especie conocida de Thomasomys. También es una de las especies de Thomasomys más grandes, superado en tamaño solo por T. apeco. Un análisis filogenético recuperó esta especie dentro de un grupo "aureus" no monofilético con una distancia genética entre $5.47 \%$ (con T. auricularis) y $10.17 \%$ (con Thomasomys sp. 1). Además, se presenta por primera vez la posición filogenética de $T$. apeco, T. praetor y T. pyrrhonotus, lo que suscita una discusión sobre la naturaleza del grupo "aureus".

Keyworks:

Piura; Thomasomys apeco; Thomasomys praetor; Thomasomys pyrrhonotus; Yungas.

Palabras claves:

Piura; Thomasomys apeco; Thomasomys praetor; Thomasomys pyrrhonotus; Yungas.

Publicación registrada en Zoobank/ZooBank article registered

LSIDurn:Isid:zoobank.org:pub:E2B98737-FCD7-4ACE-8349-ABBBBA0894C0

Acto nomenclatural/nomenclatural act:

Thomasomys antoniobracki Ruelas \& Pacheco, 2021

LSIDurn:Isid:zoobank.org:act:633E9BAB-949B-4638-8E16-B815D3D9845B 


\section{Introduction}

The genus Thomasomys Coues, 1884 is one of the most specious genera of the subfamily Sigmodontinae comprising 46 recognized species (Pacheco 2015, Brito et al. 2019, Brito et al. 2021); however, morphological and molecular evidence suggests that diversity is underestimated (Pacheco 2003, 2015, Lee et al. 2015, Brito et al. 2019). Thomasomys is endemic to South America in tropical and subtropical habitats and distributed along the Andes in paramo, premontane, and montane forests from northern Venezuela to southern Bolivia, including isolated ranges such as the Serranía de la Macarena in Colombia, at an elevational range of about $1200 \mathrm{~m}$ to above $4500 \mathrm{~m}$ (Pacheco 2015); and isolated cordilleras like El Cóndor and Kutukú, in Ecuador (Brito et al. 2021).

The taxonomy of Thomasomys has not changed much since the last century. In the last twenty years, four new species were described, T. onkiro Luna and Pacheco, 2002 known only from the type locality in southern Peru; T. ucucha Voss, 2003 from the Cordillera Oriental at northern Ecuador; T. andersoni Salazar-Bravo and Yates, 2007 from eastern slopes of the Bolivian Andes; and T. salazari Brito, Tinoco, Curay, Vargas, Reyes-Puig, Romero and Pardiñas, 2019 from Ecuador. At the morphological level, seven informal species groups have been defined (Pacheco 2015), of which the "aureus" group includes mainly medium and the larger rodents in the genus (head and body length: 135-238 $\mathrm{mm}$ ) distributed throughout the montane forests from Venezuela and Colombia to southeastern Bolivia. Pacheco in 2015, presented several morphological traits to differentiate members of this group, such as long and soft dorsal pelage generally brown-reddish; hypothenar and thenar pad of pes level or overlapping; long digit I of pes; very long digit V; genal 1 vibrissae present; very long mystacial vibrissae; and the moderately counter-shaded body pelage pattern; narrow and convergent interorbital region, not beaded; a large and long postglenoid foramen; molar-bearing portion of the maxillae rectangular; the anteroloph labially and posteriorly oriented; anteroloph in M1 labially and posteriorly oriented; M1 paraloph oriented backward to the mesoloph; the molar hypoflexus broad; and lower molars with a distinct anteromedian flexid and broad and curved posteroflexids. However, the "aureus" group was not yet tested by other sets of characters. At the molecular level, this group was represented by few species (e.g., Salazar-Bravo \& Yates 2007a, Lee et al. 2015, 2018a, Brito et al. 2019).

Pacheco et al. (2009) recognized eighteen species of Thomasomys for Peru, of which five correspond to the "aureus" group: T. apeco Leo and Gardner, 1993, T. aureus (Tomes, 1860), T. praetor (Thomas, 1900), T. pyrrhonotus Thomas, 1886, and T. rosalinda Thomas and St. Leger, 1926. Pacheco (2003) also suggested one undescribed species (Thomasomys sp. 1) from southern Peru and Bolivia and another from southern Bolivia (Thomasomys sp. 2). Recently Brito et at. (2019) recovered two distinct clades in "aureus" group, using sequences of the cytochrome gene, one clade containing T. aureus, T. au- ricularis Anthony, 1923, and three undescribed species from Ecuador and Bolivia, and another clade containing T. aureus from Cusco, Peru (TU03540) close to T. ladewi Anthony, 1926; but their representatives of the "aureus" group were very limited. Recently, Brito et al. (2021) described one of the Ecuadorean undescribed species, suggested that T. aureus is a complex species, and recovered different topologies for the "aureus" group.

As part of an ongoing study on the taxonomy and biogeography of small mammals from the Peruvian Andes, we reviewed specimens of Thomasomys of the Museo de Historia Natural, Universidad Nacional Mayor de San Marcos and found some specimens of Thomasomys sp. from northern Peru, Piura Department, with large body-size, long white tail, and very long mystacial vibrissae. These characteristics indicate this form differed strikingly from any other congeneric species. This finding prompted further comparisons with members of the "aureus" group from Peru and Ecuador to test its distinctiveness. In this work, Thomasomys sp. nov. of the "aureus" group is described based on a combined approach using morphological and molecular techniques. Our results also allow us to discuss the nature of the "aureus" group.

\section{Material and methods}

Specimens examined. Thomasomys sp. nov. was represented by four specimens collected in Carmen de la Frontera, Huancabamba Province, Piura Department. For comparative purposes, we included three specimens of T. apeco, 12 of T. aureus sensu stricto, eight of T. praetor, four of T. pyrrhonotus, and eight of Thomasomys sp. 1 sensu Pacheco (2003). Specimens are housed in the Museo de Historia Natural, Universidad Nacional Mayor de San Marcos (MUSM, Lima) and the American Museum of Natural History (AMNH, New York). Localities are mapped and listed in Figure 1 and Appendix 1, respectively.

Morphological methods. Terminology and definitions employed followed Carleton and Musser (1989) and Voss (1988) for general features, Reig (1977) and Carleton and Musser (1989) for teeth morphology nomenclature, and Pacheco $(2003,2015)$ for anatomical features of Thomasomyini. Description of fur coloration follows Smithe (1975). External measurements (TL: total length, T: tail length, F: hindfoot length, E: ear length) and weight $(\mathrm{W})$ were transcribed from specimen labels. External measurements were taken in millimeters (mm) and mass in grams (g). All cranial and mandibular variables were measured using a dial caliper (error: \pm 0.1 $\mathrm{mm}$ ). We took 21 measurements following Luna \& Pacheco (2002):

- Greatest length of skull (GLS), measured from the tip of the nasals to the posterior margin of occiput.

- Condyloincisive length (CIL), distance from the greater curvature of an upper incisor to the articular surface of the occipital condyle on the same side.

- Condylomolar length (CML), distance from the anterior edge of the first upper molar to the articular surface of the occipital condyle on the same side. 
- Length of orbital fossa (LOF), greatest distance of the orbital fossa inside the maxillary and squamosal roots of the zygomatic arch.

- Length of nasals (LN), the greatest length of either nasal bone.

- Diastema length (LD), measured from the crown of the first upper molar to the exposed lesser curvature of the upper incisor on the same side.

- Length of incisive foramina (LIF), distance from the anterior to the posterior edge of one incisive foramen.

- Length of maxillary toothrow (LM), occlusal length of the upper molar row.

- Breadth of incisive foramina (BIF), the greatest distance across the incisive foramina.

- Breadth of rostrum (BR), the least breadth between the anteroventral edges of the zygomatic plates.

- Breadth of palatal bridge (BPB), measured between the protocones of first maxillary molars.

- Breadth of first upper molar (BM1), measured across the protocone-paracone cusp pair of the first upper molar.

- Breadth of nasals (BN), the greatest distance across both nasal bones.

- Least interorbital breadth (LIB), the least interorbital breadth.

- Zygomatic breadth (ZB), the greatest distance across the zygomatic arches.

- Rostral length (RL), from zygomatic notch to the tip of the nasal bone.

- Braincase breadth (BB), measured immediately posterodorsal to the squamosal roots of the zygomatic arches.

- Breadth of zygomatic plate (BZP), the distance between the anterior and posterior edges of the zygomatic plate.

- Depth of incisor (DI), distance between greater and lesser curvature of the upper incisor.

- Height of braincase (HBC), distance from the top of the braincase to the ventral surface of the basisphenoid and basioccipital bones.

- Mesopterygoid fossa breadth (MFB), greater width of the fossa.

- Length of mandibular toothrow (MN), occlusal length of the lower molar row.

Differences in proportions of the skull measures were illustrated by a ratio diagram to show the proportional relationships among the analyzed taxa, following Musser (1970). Values of the Thomasomys sp. nov. were used as the midpoint of the standard respect the other species, large measurements than the standard are represented by positive values, those smaller by negative values. For this analysis, we used R (R Development Core Team) with the interface Rstudio 1.2.5001 (RStudio Team 2020) and the "broom" (Robinson et al. 2021), "tidyverse" (Wickham 2019), "readr" (Wickham \& Hester 2020), "dplyr" (Wickham et al. 2021), and "scales" (Wickham \& Seidel 2020) packages. Statistic methods were not performed because of the small sample size of cleaned skulls of Thomasomys sp. nov. $(\mathrm{n}=2)$. For comparative purposes, we added measurements of T. pardignasi from Brito et al. (2021).

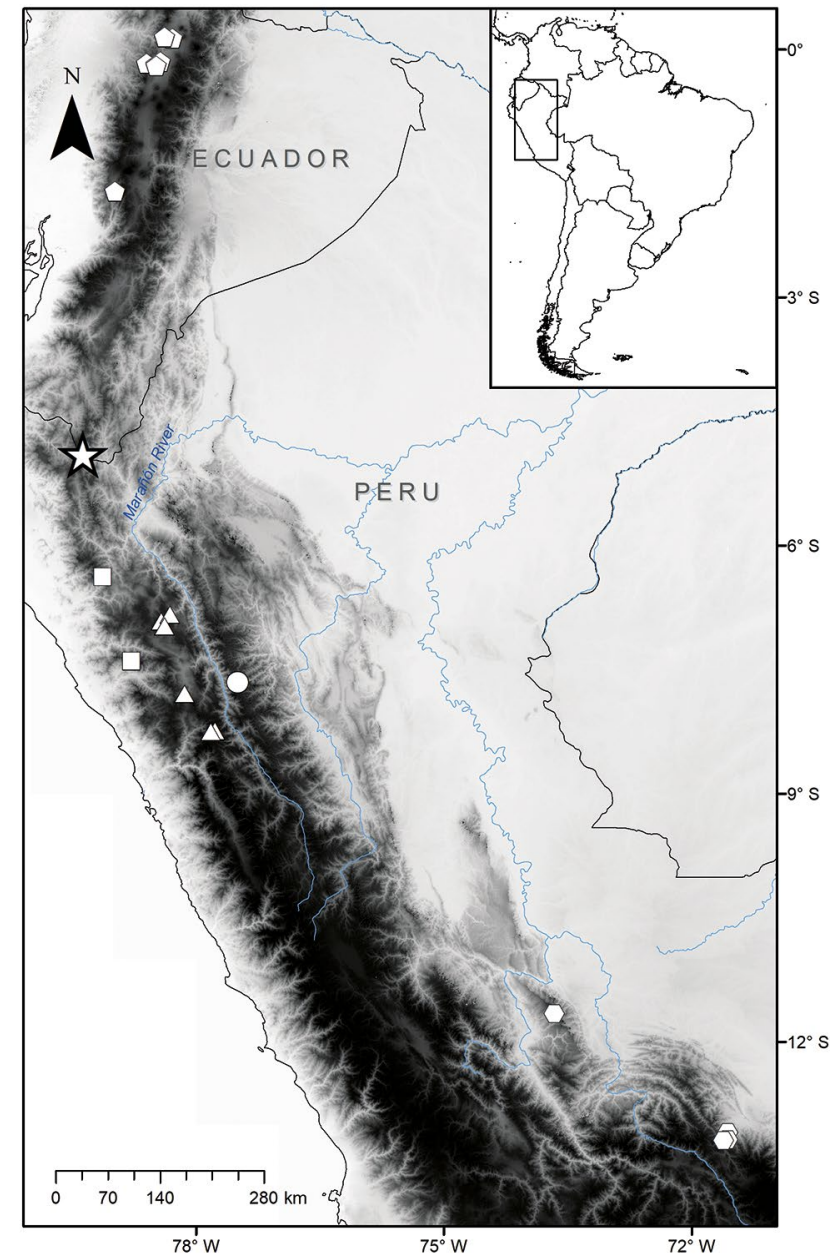

Figure 1. Map showing the collecting localities of Thomasomys antoniobracki sp. nov. (star), T. apeco (circle), T. aureus (pentagons), T. praetor (triangles), T. pyrrhonotus (squares), and Thomasomys sp. 1 (hexagons). Localities are listed in Appendix 1.

DNA extraction, amplification, and sequencing. We isolated whole DNA from dry skins of two museum specimens of Thomasomys sp. nov. from Piura using the Vivantis extraction kit (Vivantis Technologies, Selangor Darul Ehsan, Malaysia). Also, we isolated DNA from liver or muscle samples preserved in $90 \%$ ethanol from two specimens of Thomasomys sp.1, three of T. apeco, three of T. praetor, and three of T. pyrrhonotus. We amplified mitochondrial cytochrome b (Cytb) using the primers MVZ05 and MVZ16 (Smith \& Patton 1993). PCR products were sequenced by Macrogen (Macrogen Inc., Korea). We checked the sequences using CodonCode Aligner 6.0.2 (CodonCode Corporation, Dedham, USA) and deposited them in Genbank.

Phylogenetic analysis. We reconstructed the phylogeny, including the obtained sequences and others downloaded from Genbank from prior publications. We used Sigmodon hispidus, Abrothrix olivaceus, Andinomys edax, Phyllotis magister, Auliscomys micropus, Wiedomys pyrrhor- 
hinos, Oecomys bicolor, Microryzomys minutus, Oxymycterus rufus, Akodon boliviensis, Rhagomys longilingua, Rhipidomys latimanus, Rhipidomys mastacalis, Chilomys instans, and species of other Thomasomys's groups (except "aureus" group) as the outgroup, and Thomasomys sp. from Piura and species of "aureus" group as the ingroup. All sequences used are listed in Appendix 2. Sequences were aligned by Clustal in MEGA 7.0.14 (Kumar et al. 2016). Substitution model $\mathrm{TrN}+\mathrm{I}+\mathrm{G}$ was selected with jModelTest 2.1.7 (Darriba et al. 2012) by AIC using the "propose model" routine. We performed Maximum Likelihood (ML) and Bayesian inference (BI) methods to reconstruct the phylogenetic relationships of the "aureus" group. The ML analysis was performed by 10000 non-parametric bootstrap replicates to find the best tree using IQ-TREE (Nguyen et al. 2015). Bootstrap values were used as a branch support measure. For the BI analysis, two independent runs were performed with four Markov chains and 10 million iterations; the trees were sampled every 1000 generations, with the first $25 \%$ samples from the cold chain (relburnin = yes and burninfrac $=0.25$ ) discarded by burn-in (Ronquist et al. 2009). This procedure was performed using MrBayes 3.2 (Ronquist et al. 2012) using the GTR $+\mathrm{I}+\mathrm{G}$ model. Phylogenetic trees were edited in iTOL (Letunic \& Bork 2016). Convergence to stable values was checked using Tracer v1.7 (Rambaut et al. 2018) to obtain effective sample sizes (ESS) greater than 200 for all parameters. Finally, uncorrected genetic distances were estimated using the p-distances with 10000 bootstraps replicates from the aligned sequences matrix using Mega 7.0.14.

\section{Results}

Comparisons. Thomasomys sp. nov. is readily separated from any other species of Thomasomys by absolute size (see Table 1) and external and craniodental traits. We provide comparisons with some species of the "aureus" group, which could be potentially sympatric.

Compared to Thomasomys apeco, Thomasomys sp. nov. has ochraceous-tawny dorsal fur versus raw sienna to ochraceous-tawny; dorsal band is present; tail is entirely whitish versus whitish only in the distal half in $T$. apeco; mystacial vibrissae are conspicuously longer than ears while in T. apeco these vibrissae are only slightly longer; metatarsal patch is present (versus absent); ungual tufts are more abundant and longer; hairs on the rump are less dense and shorter (13.00 mm versus $19.30 \mathrm{~mm}$ ) (Table 2, Figs. 2 and 3). Cranially, rostrum is narrower; nasals are narrower, tapering with anterior margin angular (versus spoon-like with anterior margin rounded); nasals are longer extending beyond the maxillo-fronto-lacrimal (MFL) joint (versus short, anterior to MFL joint); lacrimals are larger; petrosal bone is perforated (versus no perforated); hamular process is straight (versus curved); palatal process is smaller; auditive bullae is moderately inflated (versus no inflated); foramen ovale is larger; stapedial spine is longer; coronoid process is triangular-shaped while in T. apeco is sigmoid-shaped; sigmoid notch is angular while in T. apeco is rounded; M1 protoloph is present (Table 2, Figs. 4 and 5).
Compared to Thomasomys aureus s.s., Thomasomys sp. nov. has ochraceous-tawny dorsal fur versus grizzled golden-brown; dorsal band is present; hairs on the hindfeet are dark brown versus whitish in T. aureus; tail is whitish while in T. aureus is darkish; ventral is yellowish less intense; metatarsal patch is present (Table 2, Figs. 2 and 3). Cranially, nasals are long, posterior to MFL joint (versus short, anterior to MFL joint); palatal process is small while in T. aureus s.s. it can be absent or small; sphenopalatine vacuities are slits while in T. aureus they can be closed or slits; stapedial spine is longer; coronoid process is triangular-shaped (versus sigmoid-shaped); sigmoid notch is angular (versus rounded); capsular process have more a distinct ridge and sulcus (Table 2).

Compared to Thomasomys praetor, Thomasomys sp. nov. has ochraceous-tawny dorsal fur versus olivaceous grayish; dorsal band is present; hairs on the hindfeet are dark brown (versus whitish); tail is whitish while in T. praetor is darkish; auricular patch is absent (versus present); mystacial vibrissae pass ears while in $T$. praetor pass ears slightly; tips of the ventral fur color are yellowish (versus whitish); metatarsal patch is present (versus absent); hairs on the rump are longer (13.00 $\mathrm{mm}$ versus $10.30 \mathrm{~mm}$ ); rostrum is narrower (Table 2, Figs. 2 and 3). Cranially, nasals are narrower, tapering (versus spatulated), with anterior margin angular (versus rounded); zygomatic notch is shallower; lacrimals are larger; parietal lateral process is smaller; petrosal bone is perforated (versus no perforated); mesopterygoid fossa is broader and slightly expanded anteriorly (versus narrow and parallel); incisive foramina are longer; palatal process is small (versus absent); sphenopalatine vacuities are narrow slits while in T. praetor are a little larger; stapedial spine is longer; coronoid process is triangular-shaped (versus sigmoidshaped); sigmoid notch is angular (versus rounded); capsular process has distinct ridge and sulcus (versus ridge and weak sulcus); upper molar rows are parallel while in T. praetor are divergent; M1 protoloph is present (versus absent); M1 anteromedian flexus is distinct (versus shallow); M1 mesoloph is distinct (versus short and weak) (Table 2, Figs. 4 and 5).

Compared to Thomasomys pyrrhonotus, Thomasomys sp. nov. has ochraceous-tawny dorsal fur versus rich rufous and olivaceous; dorsal band is present; hairs on the hindfeet are dark brown (versus beige); tail is whitish while in T. pyrrhonotus is darkish; mystacial vibrissae pass ears while in T. pyrrhonotus slightly pass the ears; tips of the ventral fur are yellowish (versus whitish); metatarsal patch is present while in T. pyrrhonotus is variable; hair on the rump is less dense and shorter (13.00 mm versus $11.80 \mathrm{~mm}$ ) (Table 2, Figs. 2 and 3). Cranially, anterior margin of the nasals is angular (versus rounded); nasals are long, posterior to MFL joint (versus slightly long, at level MFL joint); lacrimals are larger; parietal lateral process is smaller; hamular process is shorter; mesopterygoid fossa is broader and slightly expanded anteriorly (versus parallel); palatal process is small (versus absent); sphenopalatine vacuities are narrow (versus moderate); eustachian tube is 
slightly larger (versus short); auditive bullae is less inflated versus notably inflated; stapedial spine is longer; coronoid process is triangular-shaped versus (sigmoidshaped); sigmoid notch is angular (versus rounded); up- per molar rows are parallel while in T. pyrrhonotus are divergent; upper incisors are slightly opisthodont (versus opisthodont); M1 protoloph is present (Table 2, Figs. 4 and 5); procingulum is larger.

Table 1. Weight (in grams) and measurements (in millimeters) of adult specimens of Thomasomys antoniobracki sp. nov. (MUSM 23756, 23757), T. apeco, T. aureus, T. pardignasi, T. praetor, T. pyrrhonotus, and Thomasomys sp. 1 sensu Pacheco (2003). Values of T. aureus were taken from Pacheco (2021) and of T. pardignasi from Brito et al. (2021). Specimens are listed in Appendix 1. See abbreviations in Material and methods. Values shown above: mean, standard deviation, and sample size in parenthesis: below: minimum and maximum range.

\begin{tabular}{|c|c|c|c|c|c|c|c|c|}
\hline Measure. & $\begin{array}{l}\text { MUSM } \\
23756\end{array}$ & $\begin{array}{l}\text { MUSM } \\
23757\end{array}$ & T. apeco & T. aureus & T. pardignasi & T. praetor & T. pyrrhonotus & $\begin{array}{c}\text { Thomasomys } \\
\text { sp.1 }\end{array}$ \\
\hline W & 70 & 154 & - & - & $68-63$ & $\begin{array}{c}112.86 \pm 12.62(7) \\
92-127\end{array}$ & $\begin{array}{c}40.5 \pm 80.89(4) \\
80-94\end{array}$ & - \\
\hline $\mathrm{TL}$ & 422 & 440 & - & $\begin{array}{c}391.5 \pm 22(4) \\
360-408\end{array}$ & $371-347$ & $\begin{array}{c}361.75 \pm 26.22(8) \\
323.2-416\end{array}$ & $\begin{array}{c}329.50 \pm 17.82(4) \\
315-355\end{array}$ & - \\
\hline $\mathrm{T}$ & 238 & 252 & - & $\begin{array}{c}220 \pm 14.1(4) \\
200-233\end{array}$ & $226-210$ & $\begin{array}{c}199.25 \pm 11.95(8) \\
184-213 .\end{array}$ & $\begin{array}{c}190 \pm 7.07(4) \\
185-200\end{array}$ & - \\
\hline $\mathrm{F}$ & 41 & 42 & - & $\begin{array}{c}37.9 \pm 1.8(15) \\
35-41\end{array}$ & $35-38$ & $\begin{array}{c}35.31 \pm 1.53(8) \\
33-37\end{array}$ & $\begin{array}{c}31 \pm 0.82(4) \\
30-32\end{array}$ & - \\
\hline$E$ & 26 & 30 & - & - & $23-21$ & $\begin{array}{c}23.94 \pm 2.43(8) \\
21-29\end{array}$ & $\begin{array}{c}22.38 \pm 2.43(4) \\
20-25.5\end{array}$ & - \\
\hline GLS & - & 45.39 & $\begin{array}{c}50.41 \pm 0.30(3) \\
50.07-50.63\end{array}$ & $\begin{array}{c}40.10 \pm 1.00(11) \\
38.10-41.70\end{array}$ & - & $\begin{array}{c}40.58 \pm 0.85(7) \\
39.30-41.61 \\
\end{array}$ & $\begin{array}{c}36.64 \pm 0.27(4) \\
36.32-36.89 \\
\end{array}$ & $\begin{array}{l}38.52 \pm 0.5(8) \\
37.61-39.21 \\
\end{array}$ \\
\hline $\mathrm{CIL}$ & 40.45 & 41.35 & $\begin{array}{c}46.79 \pm 0.50(3) \\
46.29-47.28\end{array}$ & $\begin{array}{c}36.90 \pm 1.10(12) \\
35.40-39.50\end{array}$ & $33.10-32.21$ & $\begin{array}{l}37.48 \pm 0.79(8) \\
36.38-38.46\end{array}$ & $\begin{array}{l}33.89 \pm 0.50(4) \\
33.51-34.60\end{array}$ & $\begin{array}{c}35.28 \pm 0.61(8) \\
34.45-36.28\end{array}$ \\
\hline $\mathrm{CML}$ & 26.34 & 26.66 & $\begin{array}{c}30.67 \pm 0.38(3) \\
30.23-30.93\end{array}$ & $\begin{array}{c}24.1 \pm 0.8(13) \\
22.6-25.9\end{array}$ & - & $\begin{array}{c}24.27 \pm 0.61(8) \\
23.35-25.18 \\
\end{array}$ & $\begin{array}{c}22.82 \pm 0.32(4) \\
22.42-23.18 \\
\end{array}$ & $\begin{array}{c}23.10 \pm 0.35(8) \\
22.29-23.33 \\
\end{array}$ \\
\hline LOF & 15.02 & 14.68 & $\begin{array}{c}16.41 \pm 0.15(3) \\
16.32-16.58\end{array}$ & $\begin{array}{c}13.00 \pm 0.50(18) \\
12.30-14.10\end{array}$ & $12.22-11.20$ & $\begin{array}{l}13.52 \pm 0.49(8) \\
12.72-14.17\end{array}$ & $\begin{array}{c}12.37 \pm 0.47(4) \\
11.84-12.78\end{array}$ & $\begin{array}{c}12.59 \pm 0.28(8) \\
12.28-12.95\end{array}$ \\
\hline LN & 16.54 & 17.91 & $\begin{array}{c}18.86 \pm 0.17(3) \\
18.71-19.05\end{array}$ & 14.70 & $13.47-12.27$ & $\begin{array}{c}16.23 \pm 0.42(8) \\
15.7-16.92\end{array}$ & $\begin{array}{c}13.89 \pm 0.31(4) \\
13.42-14.10\end{array}$ & $\begin{array}{c}14.61 \pm 0.40(8) \\
14.15-15.30\end{array}$ \\
\hline LD & 11.87 & 12.25 & $\begin{array}{c}13.70 \pm 0.41(3) \\
13.25-14.06\end{array}$ & $\begin{array}{c}9.90 \pm 0.60(2) \\
9.40-10.30\end{array}$ & $9.10-8.82$ & $\begin{array}{c}10.91 \pm 0.30(8) \\
10.56-11.38\end{array}$ & $\begin{array}{c}9.01 \pm 0.10(4) \\
8.89-9.13\end{array}$ & $\begin{array}{c}10.1 \pm 0.59(8) \\
9.25-10.92\end{array}$ \\
\hline LIF & 9.78 & 10.11 & $\begin{array}{c}11.03 \pm 0.06(3) \\
10.99-11.10\end{array}$ & $\begin{array}{c}7.70 \pm 0.60(3) \\
7.10-8.20\end{array}$ & $6.60-6.51$ & $\begin{array}{c}8.93 \pm 0.25(8) \\
8.56-9.30\end{array}$ & $\begin{array}{c}7.75 \pm 0.04(4) \\
7.71-7.79 \\
\end{array}$ & $\begin{array}{c}8.12 \pm 0.33(8) \\
7.75-8.72\end{array}$ \\
\hline LM & 8.28 & 8.21 & $\begin{array}{c}9.60 \pm 0.26(3) \\
9.34-9.85 \\
\end{array}$ & $\begin{array}{c}7.20 \pm 0.20(3) \\
7.00-7.40\end{array}$ & $6.40-6.60$ & $\begin{array}{c}7.10 \pm 0.22(8) \\
6.67-7.40\end{array}$ & $\begin{array}{c}7.15 \pm 0.10(4) \\
7.04-7.29\end{array}$ & $\begin{array}{c}6.97 \pm 0.27(8) \\
6.62-7.38 \\
\end{array}$ \\
\hline BIF & 3.18 & 3.22 & $\begin{array}{c}3.54 \pm 0.23(3) \\
3.27-3.68 \\
\end{array}$ & $\begin{array}{c}2.70 \pm 0.20(3) \\
2.50-2.90\end{array}$ & $2.45-2.37$ & $\begin{array}{c}3.04 \pm 0.10(8) \\
2.88-3.15 \\
\end{array}$ & $\begin{array}{c}2.78 \pm 0.12(4) \\
2.65-2.93 \\
\end{array}$ & $\begin{array}{c}2.69 \pm 0.09(8) \\
2.60-2.86\end{array}$ \\
\hline $\mathrm{BR}$ & 7.46 & 7.65 & $\begin{array}{c}8.80 \pm 0.22(3) \\
8.61-9.04\end{array}$ & $\begin{array}{c}6.00 \pm 0.30(3) \\
5.70-6.20\end{array}$ & $5.81-6.24$ & $\begin{array}{c}7.15 \pm 0.28(8) \\
6.73-7.50\end{array}$ & $\begin{array}{c}6.48 \pm 0.11(4) \\
6.33-6.58\end{array}$ & $\begin{array}{c}6.44 \pm 0.27(8) \\
6.12-6.82\end{array}$ \\
\hline BPB & 3.73 & 3.81 & $\begin{array}{c}4.38 \pm 0.38(3) \\
4.02-4.77\end{array}$ & $\begin{array}{c}3.10 \pm 0.30(3) \\
2.70-3.40\end{array}$ & & $\begin{array}{c}3.06 \pm 0.15(8) \\
2.92-3.36\end{array}$ & $\begin{array}{c}2.66 \pm 0.23(4) \\
2.44-2.96 \\
\end{array}$ & $\begin{array}{c}3.14 \pm 0.16(8) \\
2.98-3.44\end{array}$ \\
\hline BM1 & 2.38 & 2.48 & $\begin{array}{c}2.69 \pm 0.08(3) \\
2.61-2.77\end{array}$ & $2.10(3)$ & $1.90-1.92$ & $\begin{array}{c}2.21 \pm 0.09(8) \\
2.09-2.32\end{array}$ & $\begin{array}{c}2.00 \pm 0.08(4) \\
1.91-2.10\end{array}$ & $\begin{array}{c}2.15 \pm 0.05(8) \\
2.06-2.21\end{array}$ \\
\hline $\mathrm{BN}$ & 5.16 & 5.07 & $\begin{array}{c}6.18 \pm 0.25(3) \\
5.90-6.38\end{array}$ & 4.30 & - & $\begin{array}{c}5.33 \pm 0.23(8) \\
4.86-5.60\end{array}$ & $\begin{array}{c}4.43 \pm 0.14(4) \\
4.29-4.60\end{array}$ & $\begin{array}{c}4.42 \pm 0.11(8) \\
4.23-4.51\end{array}$ \\
\hline LIB & 5.32 & 5.53 & $\begin{array}{c}5.10 \pm 0.23(3) \\
4.96-5.37\end{array}$ & $\begin{array}{c}5.30 \pm 0.10(3) \\
5.20-5.40\end{array}$ & $5.10-5.33$ & $\begin{array}{c}4.88 \pm 0.16(8) \\
4.68-5.16\end{array}$ & $\begin{array}{c}4.40 \pm 0.13(4) \\
4.23-4.51\end{array}$ & $\begin{array}{c}4.88 \pm 0.15(8) \\
4.64-5.10\end{array}$ \\
\hline ZB & 23.01 & 23.48 & $\begin{array}{c}26.87 \pm 0.44(3) \\
26.44-27.32 \\
\end{array}$ & $\begin{array}{c}19.20 \pm 0.30(2) \\
19.00-19.40\end{array}$ & $19.50-19.50$ & $\begin{array}{l}21.22 \pm 0.77(8) \\
20.49-22.30\end{array}$ & $\begin{array}{c}19.32 \pm 0.12(4) \\
19.22-19.50\end{array}$ & $\begin{array}{c}20.42 \pm 0.37(8) \\
19.73-20.88 \\
\end{array}$ \\
\hline $\mathrm{RL}$ & 14.76 & 15.89 & $\begin{array}{c}17.13 \pm 0.20(3) \\
16.98-17.36\end{array}$ & - & $11.54-11.31$ & $\begin{array}{c}13.86 \pm 0.33(8) \\
13.37-14.27\end{array}$ & $\begin{array}{c}12.09 \pm 0.21(4) \\
11.88-12.38\end{array}$ & $\begin{array}{c}13.22 \pm 0.32(8) \\
12.71-13.75 \\
\end{array}$ \\
\hline BB & 17.29 & 17.91 & $\begin{array}{c}20.16 \pm 0.27(3) \\
19.87-20.40\end{array}$ & $\begin{array}{c}16.70 \pm 0.60(2) \\
16.30-17.10\end{array}$ & $15.55-15.38$ & $\begin{array}{c}16.22 \pm 0.22(8) \\
16.00-16.57\end{array}$ & $\begin{array}{c}14.79 \pm 0.25(4) \\
14.56-15.14\end{array}$ & $\begin{array}{l}16.2 \pm 0.19(8) \\
15.95-16.48\end{array}$ \\
\hline BZP & 4.09 & 4.25 & $\begin{array}{c}4.78 \pm 0.06(3) \\
4.72-4.83\end{array}$ & $\begin{array}{c}3.60 \pm 0.10(3) \\
3.50-3.60\end{array}$ & $3.27-3.25$ & $\begin{array}{c}3.77 \pm 0.20(8) \\
3.54-4.16\end{array}$ & $\begin{array}{c}3.32 \pm 0.09(4) \\
3.20-3.42\end{array}$ & $\begin{array}{c}3.4 \pm 0.14(8) \\
3.21-3.58\end{array}$ \\
\hline DI & 2.12 & 2.24 & $\begin{array}{c}2.62 \pm 0.07(3) \\
2.56-2.69 \\
\end{array}$ & $\begin{array}{c}1.90 \pm 0.20(2) \\
1.70-2.00\end{array}$ & $1.80-1.79$ & $\begin{array}{c}2.33 \pm 0.08(8) \\
2.23-2.49 \\
\end{array}$ & $\begin{array}{c}2.13 \pm 0.17(4) \\
1.93-2.27 \\
\end{array}$ & $\begin{array}{c}2.02 \pm 0.11(8) \\
1.88-2.27\end{array}$ \\
\hline $\mathrm{HBC}$ & 12.66 & 12.46 & $\begin{array}{c}13.52 \pm 0.17(3) \\
13.32-13.62\end{array}$ & $11.50(2)$ & - & $\begin{array}{c}11.50 \pm 0.21(8) \\
11.29-11.82\end{array}$ & $\begin{array}{c}10.24 \pm 0.23(4) \\
10.05-10.54\end{array}$ & $\begin{array}{c}11.27 \pm 0.27(8) \\
10.90-11.58 \\
\end{array}$ \\
\hline MFW & 3.01 & 3.30 & $\begin{array}{c}3.81 \pm 0.25(3) \\
3.52-3.96\end{array}$ & - & - & $\begin{array}{c}2.74 \pm 0.14(8) \\
2.52-2.96\end{array}$ & $\begin{array}{c}2.33 \pm 0.13(4) \\
2.16-2.48\end{array}$ & $\begin{array}{c}2.73 \pm 0.21(8) \\
2.48-3.19 \\
\end{array}$ \\
\hline $\mathrm{MN}$ & 8.53 & 8.51 & $\begin{array}{l}9.92 \pm 0.29(3) \\
9.74-10.25\end{array}$ & - & $7.02-6.92$ & $\begin{array}{c}7.54 \pm 0.26(8) \\
7.10-7.88\end{array}$ & $\begin{array}{c}7.35 \pm 0.11(4) \\
7.23-7.47\end{array}$ & $\begin{array}{c}7.31 \pm 0.15(8) \\
7.12-7.53\end{array}$ \\
\hline
\end{tabular}


Table 2. Morphological differences among Thomasomys antoniobracki sp. nov., T. apeco, T. aureus, T. praetor, T. pyrrhonotus, and Thomasomys sp. 1 sensu Pacheco (2003).

\begin{tabular}{|c|c|c|c|c|c|c|}
\hline Characters & $\begin{array}{l}\text { T. antoniobracki } \\
\text { sp. nov. }\end{array}$ & T. apeco & T. aureus & T. praetor & T. pyrrhonotus & $\begin{array}{l}\text { Thomasomys } \\
\text { sp. } 1\end{array}$ \\
\hline Dorsal fur color & Ochraceous-tawny & $\begin{array}{l}\text { Raw sienna to } \\
\text { ochraceous-tawny }\end{array}$ & $\begin{array}{l}\text { Grizzled golden- } \\
\text { brown }\end{array}$ & Olivaceous grayish & $\begin{array}{l}\text { Rich rufous and } \\
\text { olivaceous }\end{array}$ & Rich rufous \\
\hline Dorsal band & Present & Absent & Absent & Absent & Absent & Present \\
\hline Hairs on the hindfeet & Dark brown & Dark brown & Whitish & Whitish & Beige & Beige \\
\hline Color tail pattern (along) & Entirely whitish & $\begin{array}{l}\text { Whitish distal half } \\
\text { and darkish basal } \\
\text { half }\end{array}$ & Entirely darkish & Entirely darkish & Entirely darkish & Entirely darkish \\
\hline Auricular patch & Absent & Absent & Absent & Present & Absent & Absent \\
\hline Mystacial vibrissae & Pass ears & Pass ears slightly & Pass ears & Pass ears slightly & Pass ears slightly & Pass ears slightly \\
\hline Ventral fur color (tips) & Yellowish & Yellowish & $\begin{array}{l}\text { Yellowish, almost } \\
\text { golden }\end{array}$ & Whitish & Whitish & Yellowish \\
\hline Metatarsal patch & Present & Absent & Absent & Absent & Sometimes present & Present \\
\hline Ungual pes tuft & $\begin{array}{l}\text { Abundant and } \\
\text { long }\end{array}$ & Scarce and short & $\begin{array}{l}\text { Abundant and } \\
\text { long }\end{array}$ & $\begin{array}{l}\text { Abundant and } \\
\text { long }\end{array}$ & $\begin{array}{l}\text { Abundant and } \\
\text { long }\end{array}$ & Scarce and long \\
\hline Hair on the rump & $\begin{array}{l}\text { No dense and } \\
\text { short }(13.00 \mathrm{~mm})\end{array}$ & $\begin{array}{l}\text { Dense and large } \\
(19.30 \mathrm{~mm})\end{array}$ & - & $\begin{array}{l}\text { No dense and } \\
\text { short }(10.30 \mathrm{~mm})\end{array}$ & $\begin{array}{l}\text { Dense and short } \\
(11.80 \mathrm{~mm})\end{array}$ & $\begin{array}{l}\text { No dense and } \\
\text { short }(10.50 \mathrm{~mm})\end{array}$ \\
\hline Rostrum & Narrow & Broad & Narrow & Broad & Narrow & Narrow \\
\hline Nasal shape & $\begin{array}{l}\text { Narrow, tapering, } \\
\text { anterior margin } \\
\text { angular }\end{array}$ & $\begin{array}{l}\text { Spoon-like, anteri- } \\
\text { or margin rounded }\end{array}$ & $\begin{array}{l}\text { Narrow, tapering, } \\
\text { anterior margin } \\
\text { angular }\end{array}$ & $\begin{array}{l}\text { Broad, spatulated, } \\
\text { anterior margin } \\
\text { rounded }\end{array}$ & $\begin{array}{l}\text { Narrow, tapering, } \\
\text { anterior margin } \\
\text { rounded }\end{array}$ & $\begin{array}{l}\text { Narrow, tapering, } \\
\text { anterior margin } \\
\text { rounded }\end{array}$ \\
\hline Nasal length & $\begin{array}{l}\text { Long, posterior to } \\
\text { MFL joint }\end{array}$ & $\begin{array}{l}\text { Short, anterior to } \\
\text { MFL joint }\end{array}$ & $\begin{array}{l}\text { Short, anterior to } \\
\text { MFL joint }\end{array}$ & $\begin{array}{l}\text { Long, posterior to } \\
\text { MFL joint }\end{array}$ & Level MFL joint & Level MFL joint \\
\hline Zygomatic notch & Shallow & Shallow & Shallow & Deep & Shallow & Shallow \\
\hline Lacrimals & Large & Small & Large & Small & Small & Large \\
\hline Parietal lateral process & Small & Small & Small & Large, broad & Large, subtriangular & Small \\
\hline Petrosal bone & Perforated & No perforated & Perforated & No perforated & Perforated & Perforated \\
\hline Hamular process & $\begin{array}{l}\text { Long, narrow, } \\
\text { straight }\end{array}$ & Long, curved & $\begin{array}{l}\text { Long, narrow, } \\
\text { straight }\end{array}$ & $\begin{array}{l}\text { Long, narrow, } \\
\text { straight }\end{array}$ & $\begin{array}{l}\text { Very long and } \\
\text { narrow, straight }\end{array}$ & $\begin{array}{l}\text { Long, narrow, } \\
\text { straight }\end{array}$ \\
\hline Incisive foramina & Long & Long & Long & Moderate & Long & Long \\
\hline Mesopterygoid fossa & $\begin{array}{l}\text { Broad, slightly ex- } \\
\text { panded anteriorly }\end{array}$ & $\begin{array}{l}\text { Broad, slightly ex- } \\
\text { panded anteriorly }\end{array}$ & $\begin{array}{l}\text { Broad, slightly ex- } \\
\text { panded anteriorly }\end{array}$ & Narrow, parallel & Narrow, parallel & $\begin{array}{l}\text { Broad, slightly ex- } \\
\text { panded anteriorly }\end{array}$ \\
\hline Palatal process & Small & Distinct & Absent or small & Absent & Absent & Small \\
\hline Sphenopalatine vacuities & Slits & Slits & Closed or slit & Moderate & Moderate & Closed \\
\hline Foramen ovale & Large & Small & Large & Large & Large & Large \\
\hline Eustachian tube & Medium & Medium & Medium & Medium & Short & Medium \\
\hline Auditive bullae & $\begin{array}{l}\text { Moderately in- } \\
\text { flated }\end{array}$ & No inflated & $\begin{array}{l}\text { Moderately in- } \\
\text { flated }\end{array}$ & $\begin{array}{l}\text { Moderately in- } \\
\text { flated }\end{array}$ & Largely inflated & No inflated \\
\hline Stapedial spine & Long and narrow & Short & Short & Short & Short & Short \\
\hline Coronoid process & Triangular-shaped & Sigmoid-shaped & Sigmoid-shaped & Sigmoid-shaped & Sigmoid-shaped & Sigmoid-shaped \\
\hline Sigmoid notch & Angular & Rounded & Rounded & Rounded & Rounded & Rounded \\
\hline Capsular process & $\begin{array}{l}\text { Distinct ridge and } \\
\text { sulcus }\end{array}$ & $\begin{array}{l}\text { Distinct ridge and } \\
\text { sulcus }\end{array}$ & $\begin{array}{l}\text { Ridge and weak } \\
\text { sulcus }\end{array}$ & $\begin{array}{l}\text { Ridge and weak } \\
\text { sulcus }\end{array}$ & $\begin{array}{l}\text { Distinct ridge and } \\
\text { sulcus }\end{array}$ & $\begin{array}{l}\text { Ridge and weak } \\
\text { sulcus }\end{array}$ \\
\hline Upper molar rows & Parallel & Parallel & Parallel & Divergent & Divergent & Parallel \\
\hline Upper Incisors & $\begin{array}{l}\text { Slightly opistho- } \\
\text { dont }\end{array}$ & $\begin{array}{l}\text { Slightly opistho- } \\
\text { dont }\end{array}$ & $\begin{array}{l}\text { Slightly opistho- } \\
\text { dont }\end{array}$ & $\begin{array}{l}\text { Slightly opistho- } \\
\text { dont }\end{array}$ & Opisthodont & $\begin{array}{l}\text { Slightly opistho- } \\
\text { dont }\end{array}$ \\
\hline M1 protoloph & Present & Absent & - & Absent & Absent & Absent \\
\hline M1 anteromedian flexus & Distinct & Distinct & - & Shallow & Distinct & Distinct \\
\hline M1 mesoloph & Distinct & Distinct & - & Short, weak & Distinct & Distinct \\
\hline $\mathrm{m} 1$ procingulum & Large & Large & - & Large & Small & Small \\
\hline
\end{tabular}




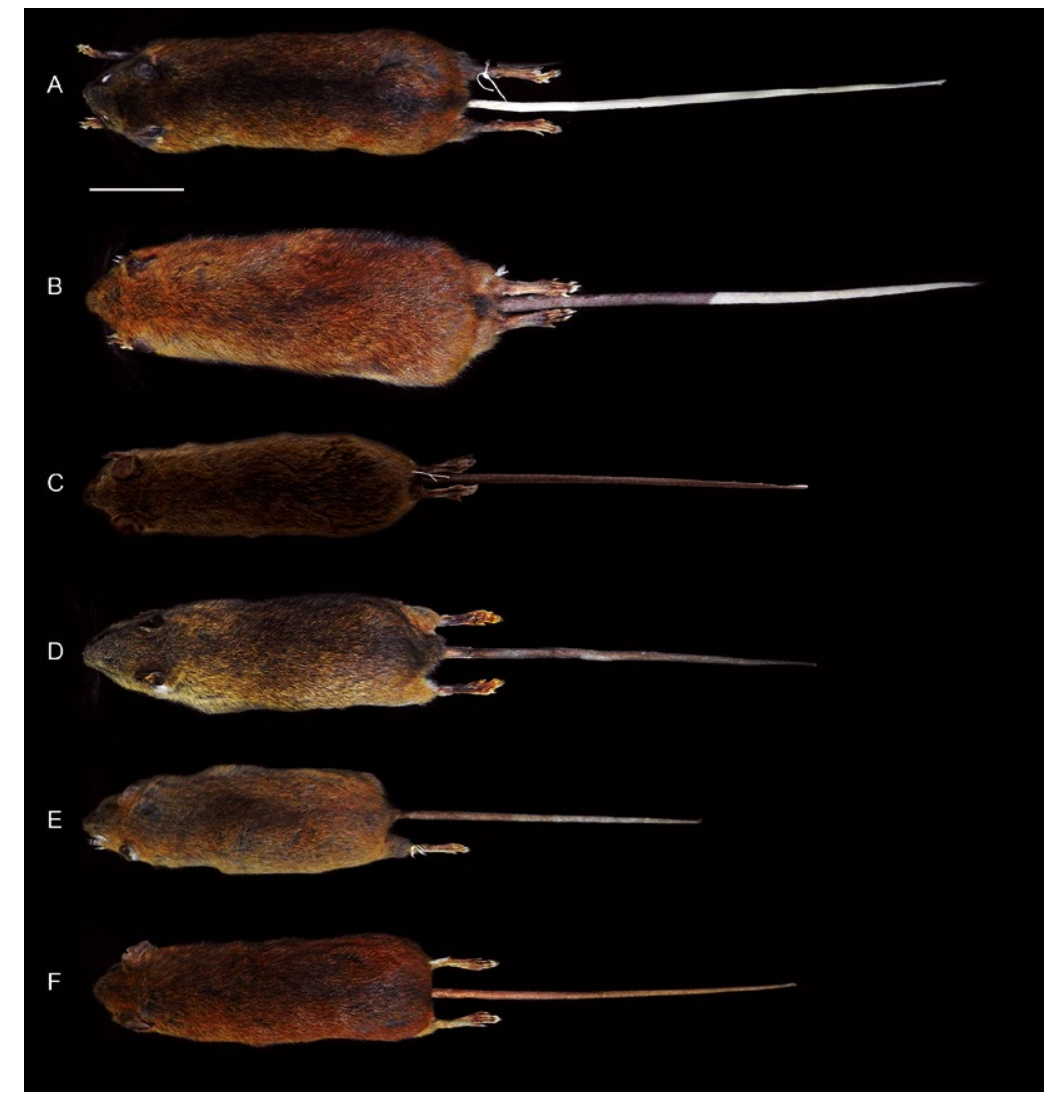

Figure 2. Dorsal views of the skin of Thomasomys antoniobracki sp. nov. (A: MUSM 23757, holotype), T. apeco (B: MUSM 7199), T. aureus (C: MSB 70707), T. praetor (D: MUSM 38498), T. pyrrhonotus (E: MUSM 46761), and Thomasomys sp. 1 (F: MUSM 9347). Scale: 50 mm.

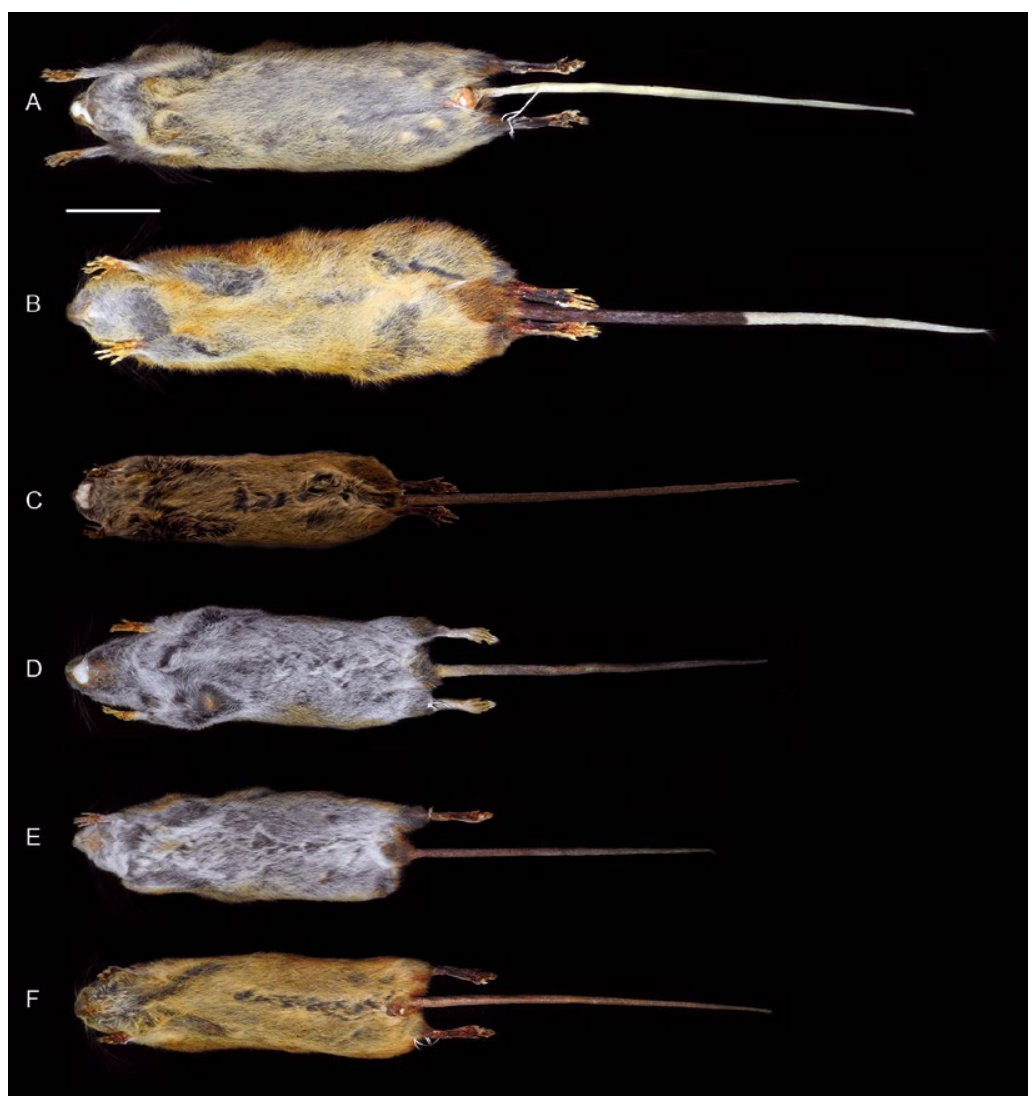

Figure 3. Ventral views of the skin of Thomasomys antoniobracki sp. nov. (A: MUSM 23757, holotype), T. apeco (B: MUSM 7199), T. aureus (C: MSB 70707), T. praetor (D: MUSM 38498), T. pyrrhonotus (E: MUSM 46761), and Thomasomys sp. 1 (F: MUSM 9347). Scale: 50 mm. 
Compared to Thomasomys sp. 1 sensu Pacheco (2003), Thomasomys sp. nov. has ochraceous-tawny dorsal fur versus rich rufous; hairs on the hindfeet are dark brown (versus beige); tail is whitish versus darkish in Thomasomys sp.1; mystacial vibrissae are distinctly longer than ears while in Thomasomys sp. 1 are slightly longer; ungual tuft on the pes is more abundant; hairs on the rump are longer ( $13.00 \mathrm{~mm}$ versus $10.50 \mathrm{~mm}$ ) (Table 2, Figs. 2 and 3). Cranially, anterior margin of the nasal is angular versus rounded in Thomasomys sp.1; nasals are long, posterior to MFL joint (versus slightly long, at level MFL joint); sphenopalatine vacuities are narrow versus closed; auditive bullae is moderately inflated (versus no inflated); stapedial spine is longer; coronoid process is triangular-shaped while in Thomasomys sp. 1 is sigmoidshaped; sigmoid notch is angular in T. aureus is rounded; capsular process has distinct ridge and sulcus (versus ridge and weak sulcus); M1 protoloph is present; procingulum is larger (Table 2, Figs. 4 and 5).
Thomasomys sp. nov. is smaller than T. apeco in all variables, but is larger than T. aureus S.S., T. pyrrhonotus, and Thomasomys sp. 1 in all variables. Compared with T. praetor, Thomasomys sp. nov. is larger in most variables, except in BN and DI (Table 1, Fig. 6). Among analyzed species, T. pardignasi and T. pyrrhonotus were the smallest species.

Molecular evidence. The phylogenetic analysis was based on molecular data of 81 sequences of $C y t b$ gene of 801 bp of which 425 were constant, 321 were parsimony informative, and 55 were singletons. The ML and BI analysis of the $C y t b$ matrix failed to recover a monophyletic "aureus" group (Fig. 7). Thomasomys apeco and Thomasomys sp. 1, members of "aureus" group (following Pacheco (2015)) form a monophyletic group (bs = 86, pp = 0.9); however, this clade was recovered as sister of Thomasomys notatus ("notatus" group); and this clade as the sister group (bs = 79) of the clade T. baeops + T. taczanowskii ("baeops" group) (bs = 100, pp =1).
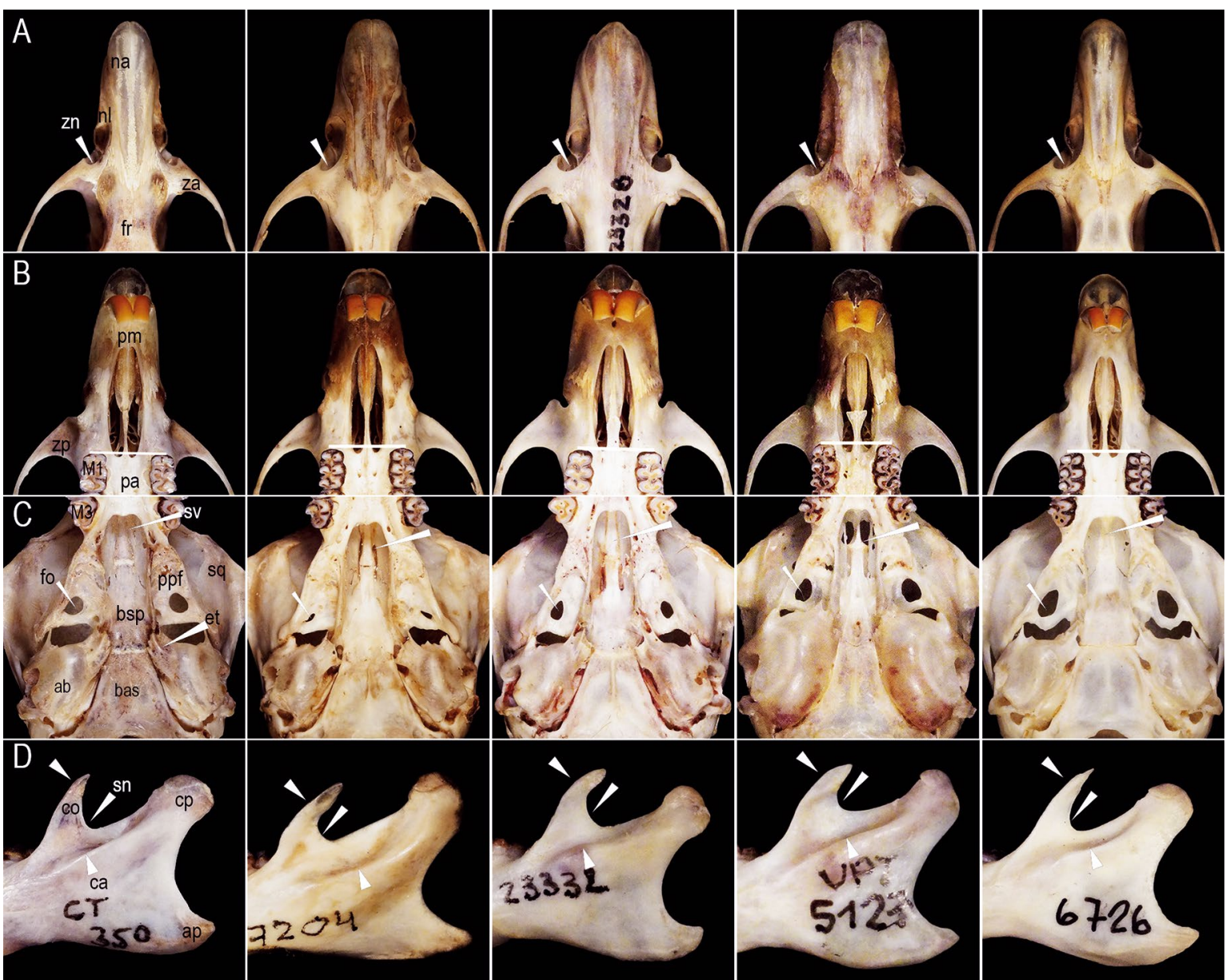

Figure 4. Morphological comparisons (from left to right) among Thomasomys antoniobracki sp. nov. (MUSM 23757, holotype), $T$. apeco (MUSM 7204), T. praetor (MUSM 23328), T. pyrrhonotus (MUSM 52410), and Thomasomys sp. 1 (MUSM 6726). Characters shown in T. antoniobracki sp. nov.: A) zygomatic notch shallow and the narrow rostrum, B) long incisive foramina, C) short palate, sphenopalatine vacuities slits, medium-sized eustachian tube, and moderately inflated auditive bullae, D) triangular-shaped coronoid process slightly short, angular sigmoid notch and distinct ridge and sulcus of the capsular process. Abbreviations: ab, auditory bulla; af, anteromedian flexus; ap, angular process; bas, basioccipital; bsp, basisphenoid; ca, capsular process; co, coronoid process; $\mathrm{cp}$, condylar process; et, eustachian tube; fo, foramen ovale; fr, frontal; M1, first upper molar; M3, third upper molar; me, mesoloph; na, nasal; nl, nasolacrimal foramen; pa, palatal; pm, premaxilar; ppf, parapterygoid fossa; pr, protoloph; sn, sigmoid notch; sq, squamosal; sv, sphenopalatal vacuities; za, zygomatic arch; zn, zygomatic notch; zp, zygomatic plate. Not scaled. 


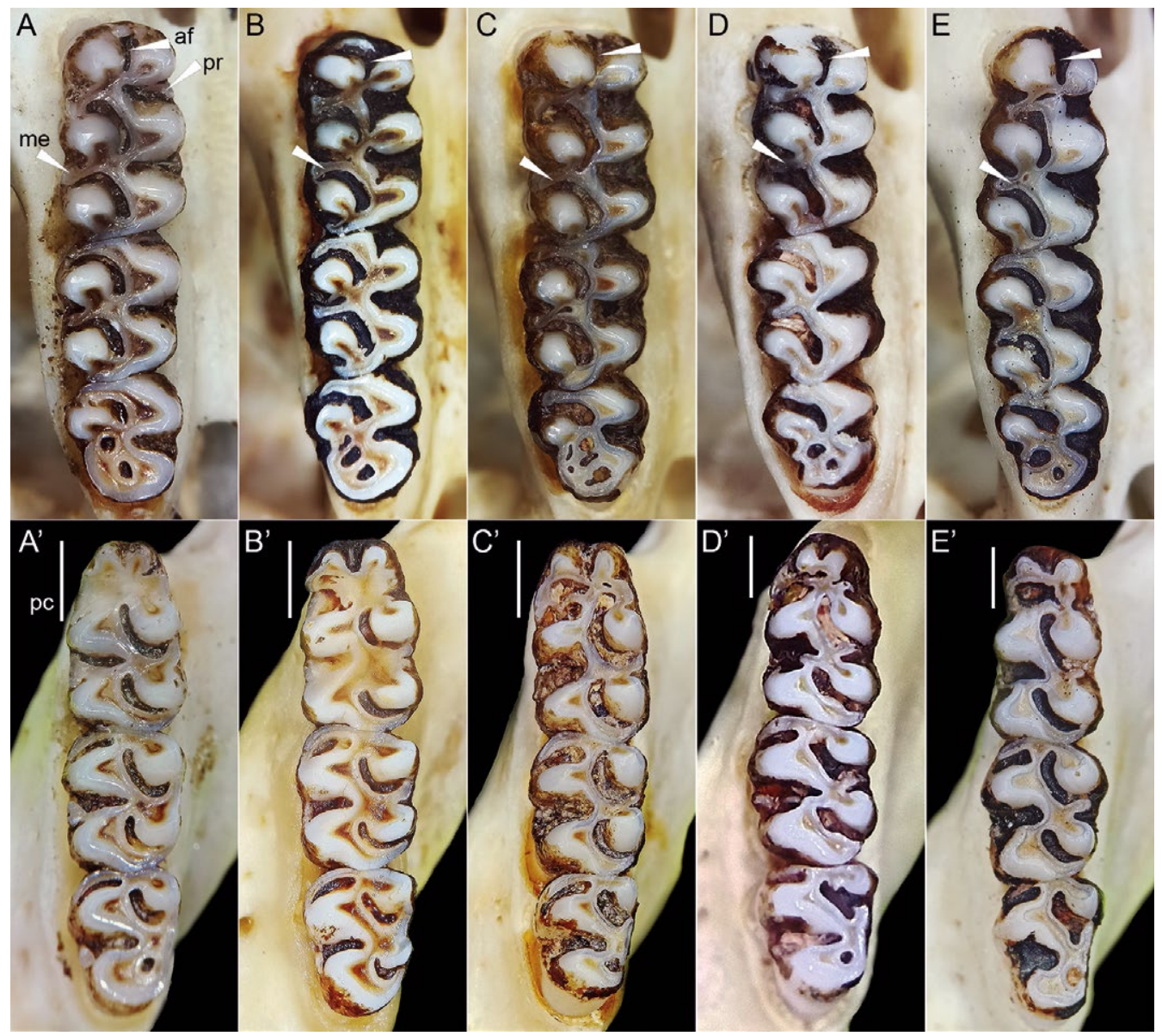

Figure 5. Comparisons of upper molars (top) and lower molars (bottom) of A) Thomasomys antoniobracki sp. nov. (MUSM 23756), B) T. apeco (MUSM 7198 [top], MUSM 7204 [bottom]), C) Thomasomys sp. 1 (MUSM 9363), D) T. praetor (MUSM 40211), and D) T. pyrrhonotus (MUSM 40359). See in Thomasomys antoniobracki sp. nov. protoloph present in first upper molar, distinct anteromedian flexus and mesoloph in first upper molar. Abbreviations: af, anteromedian flexus; me, mesoloph; pc, procingulum; pr, protoloph. Not scaled.

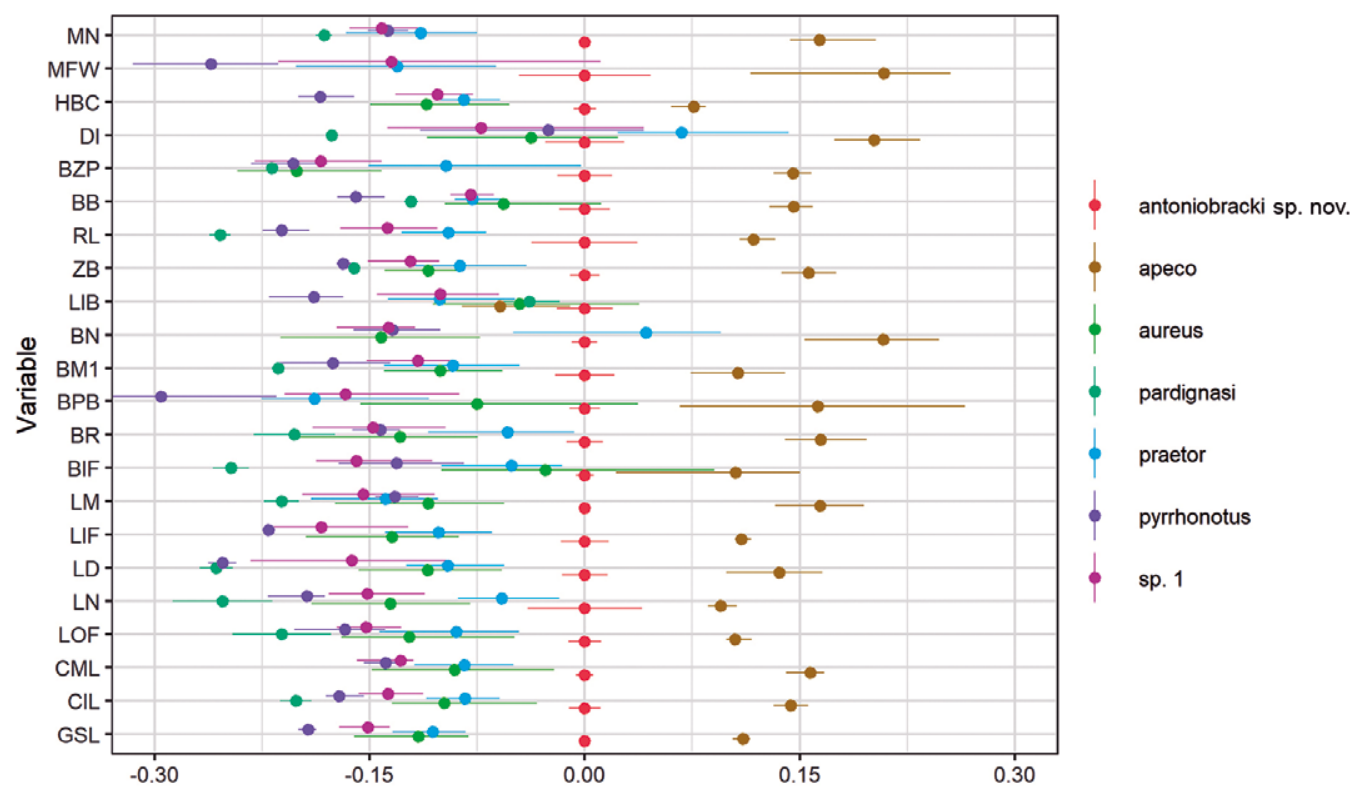

Figure 6. Ratio diagram comparing the mean values for each external and cranial dimension among T. antoniobracki sp. nov., Thomasomys apeco, T. aureus (taken from Pacheco 2021), T. praetor, T. pardignasi (taken from Brito et al. 2021), T. pyrrhonotus, and Thomasomys sp. 1. See Materials and Methods for abbreviations. 


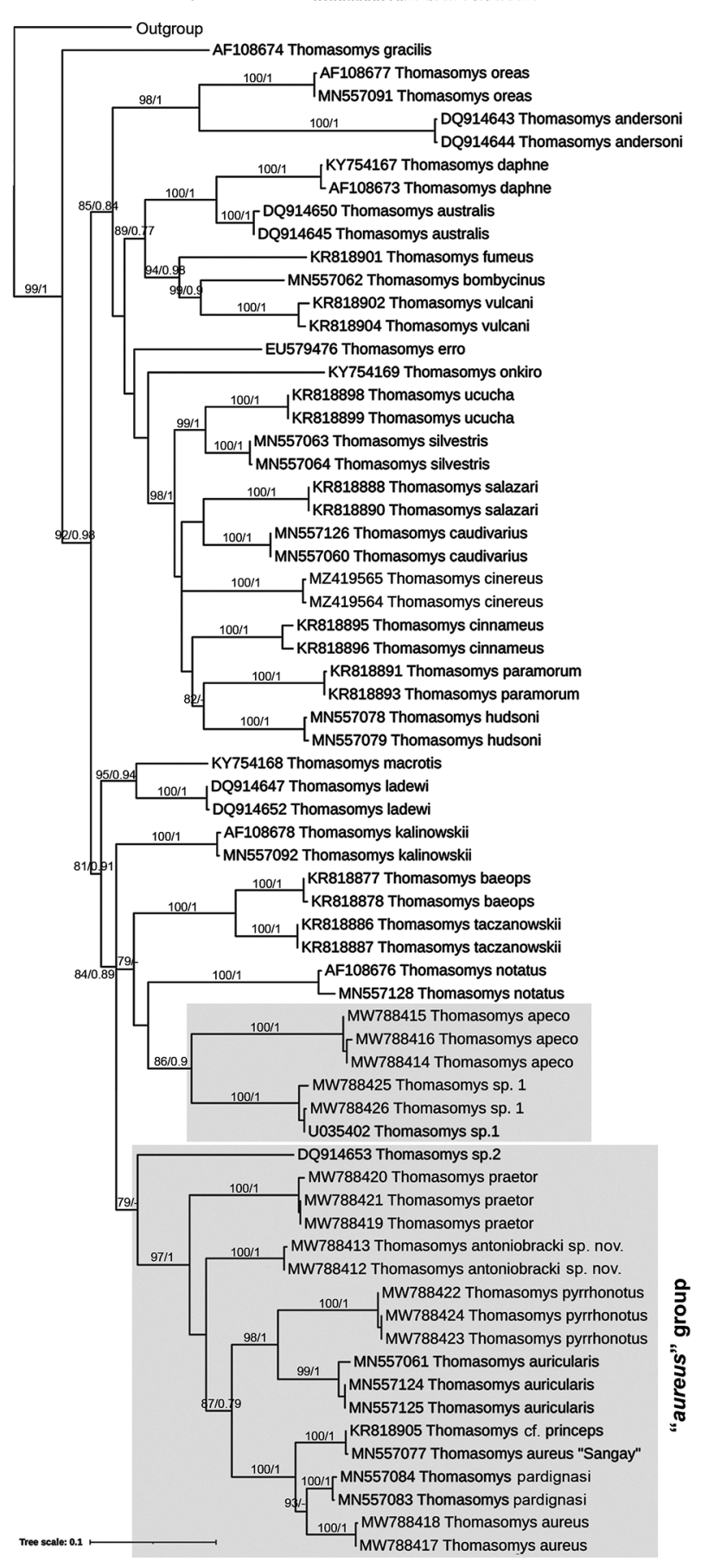

Figure 7. Tree resulting from the maximum-likelihood analysis of species of "aureus" group of Thomasomys. Numbers on the branches indicate ML bootstrap values (left of the diagonal) and Bayesian posterior probabilities (right of the diagonal). Sequences are listed in Appendix 2. The "aureus" group is highlighted in gray.

All the other studied species members of "aureus" group were recovered in a monophyletic group with moderate support $(\mathrm{bs}=79)$. Thomasomys sp. 2 . was recovered as sister of the other species. ML and BI topologies were congruent showing Thomasomys sp. nov. into a well-supported clade (bs $=97, \mathrm{pp}=1$ ) containing T. $a u$ reus s.s., T. aureus "Sangay" (MN557077), T. auricularis, $T$. pardignasi, T. praetor, T. cf. princeps, and T. pyrrhonotus. The genetic divergence observed between Thomasomys sp. nov. and the remaining species of the "aureus" group range from $5.47 \%$ (with T. auricularis) to $10.17 \%$ (with Thomasomys sp. 1) (Table 3). Intraspecific distance of Thomasomys sp. nov. is $0.51 \pm 0.29 \%$. On the other hand, the genetic distance of T. aureus s.s. versus T. aureus "Sangay" was $5.64 \pm 1.19 \%$, versus T. pardignasi was $4.49 \pm$ $1.01 \%$, versus Thomasomys sp. 1 was $11.88 \pm 1.67 \%$, and versus Thomasomys sp. 2 was $9.74 \pm 1.60 \%$ (Table 3).

Given the evidence from the morphological, morphometric, and molecular analyses, we recognize that Thomasomys sp. nov. from Piura Department as a full species and member of the "aureus" group. Because no name is available for this new taxon, we provide a name and a formal description.

\section{Taxonomy \\ ORDER RODENTIA BOWDICH, 1821 \\ FAMILY CRICETIDAE FISCHER, 1817 \\ SUBFAMILY SIGMODONTINAE WAGNER, 1843 \\ TRIBE THOMASOMYINI STEADMAN AND RAY, 1982}

\section{Thomasomys antoniobracki sp. nov.}

\section{"RATÓN MONTARAZ COLIBLANCO DE ANTONIO BRACK"}

Holotype. The holotype is an adult female specimen deposited at the Departamento de Mastozoología, Museo de Historia Natural de la Universidad Nacional Mayor de San Marcos (MUSM 23757), collected by Carlos Tello (original field number CT 350) on April 15, 2006, and prepared as skin and cleaned skull, with carcass preserved in ethanol. (Fig. 8).

Type locality. Campamento Alto Parramata at 2630 $m$ elevation, in Carmen de la frontera district, Huancabamba province, Piura department, Peru, $4.89429^{\circ} \mathrm{S}$, 79.36761ํW (Fig. 1).

Paratypes. Paratypes include three adult specimens preserved as skull, skin, and carcass (MUSM 23756) and alcoholic specimens (MUSM 23754, 23755) collected in the type locality. Measurements of the holotype and paratype specimens are provided in Table 1.

Diagnosis. A large rodent of the genus Thomasomys in the "aureus" group (head and body length: 184-188 $\mathrm{mm}$ ), distinguished from congenerics by the following combination of characters: narrow rostrum; narrow nasals, tapering and long extending to the maxillary-frontal-lacrimal intersection or beyond; shallow zygomatic notch; large lacrimals; palate bone narrow with posterior margin leveling about half M3, small palatal process present; mesopterygoid fossa broad, slightly expanded anteriorly; sphenopalatine vacuities present as narrow slits; large oval foramen; auditory bullae moderately inflated; stapedial spine long and narrow; capsular process absent but produced as a swollen ridge with an inner sulcus between the ridge and the sigmoid notch; coronoid process triangular and sigmoid notch angular; upper incisors slightly opisthodont; M1 protoloph present; M1 and M3 hypoflexus distinct. 
Table 3. Uncorrected genetic distances within (In: intraspecific distances) and among (below diagonal: p-distances, above diagonal: standard error) the species of the "aureus" group of Thomasomys.

\begin{tabular}{|c|c|c|c|c|c|c|c|c|c|c|c|c|}
\hline Species & In & 1 & 2 & 3 & 4 & 5 & 6 & 7 & 8 & 9 & 10 & 11 \\
\hline 1) antoniobracki sp. nov. & 0.51 & & 1.47 & 1.31 & 1.46 & 1.11 & 1.27 & 1.36 & 1.44 & 1.43 & 1.52 & 1.50 \\
\hline 2) apeco & 0.51 & 9.40 & & 1.55 & 1.47 & 1.63 & 1.49 & 1.56 & 1.49 & 1.51 & 1.64 & 1.54 \\
\hline 3) aureus s.s. & - & 7.69 & 10.17 & & 1.21 & 1.45 & 1.01 & 1.50 & 1.18 & 1.46 & 1.66 & 1.60 \\
\hline 4) aureus "Sangay" & - & 9.74 & 9.32 & 5.64 & & 1.61 & 1.06 & 1.63 & 0.24 & 1.55 & 1.83 & 1.66 \\
\hline 5) auricularis & 0.68 & 5.47 & 11.00 & 9.49 & 11.79 & & 1.41 & 1.44 & 1.60 & 1.42 & 1.71 & 1.53 \\
\hline 6) pardignasi & 0.77 & 7.56 & 9.79 & 4.49 & 4.74 & 9.62 & & 1.52 & 1.06 & 1.42 & 1.73 & 1.67 \\
\hline 7) praetor & 0.17 & 7.35 & 10.00 & 9.15 & 10.94 & 8.55 & 9.83 & & 1.63 & 1.48 & 1.65 & 1.60 \\
\hline 8) cf. princeps & - & 9.49 & 9.57 & 5.38 & 0.26 & 11.54 & 4.74 & 10.94 & & 1.53 & 1.81 & 1.64 \\
\hline 9) pyrrhonotus & 0.17 & 8.80 & 10.17 & 9.32 & 10.34 & 8.72 & 9.19 & 9.32 & 10.09 & & 1.83 & 1.63 \\
\hline 10) Thomasomys sp.1 & 0.51 & 10.17 & 11.45 & 11.88 & 14.27 & 12.82 & 13.38 & 11.37 & 14.02 & 14.44 & & 1.54 \\
\hline 11) Thomasomys sp.2 & - & 9.74 & 10.09 & 11.03 & 11.79 & 9.49 & 12.18 & 10.60 & 11.54 & 10.85 & 10.77 & \\
\hline
\end{tabular}

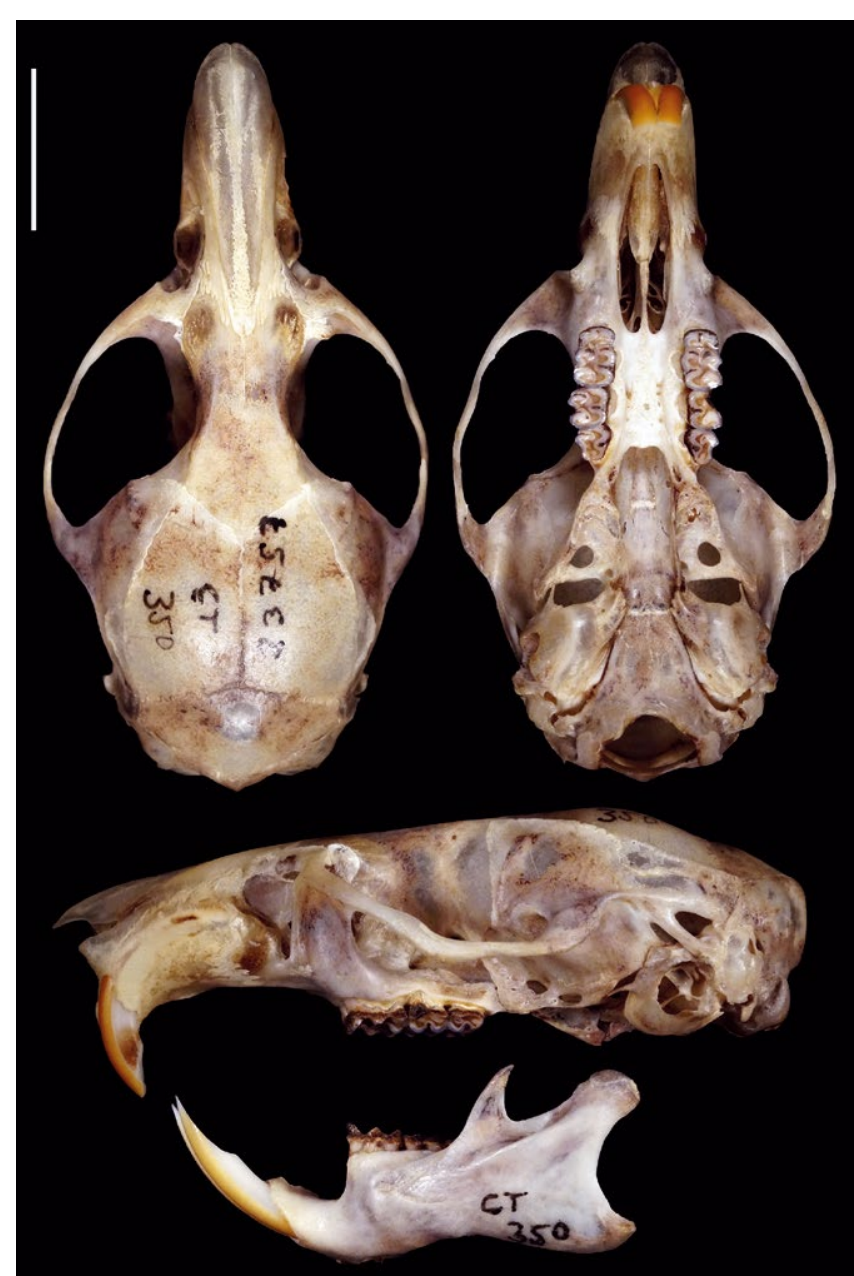

Figure 8. Dorsal, ventral, and lateral views of the cranium and lateral view of the mandible of the holotype of Thomasomys antoniobracki sp. nov. (MUSM 23757, holotype, age class 3). Scale: $10 \mathrm{~mm}$.

Description. Figs. 2, 3, 4, 5, 8, and 9. Body. Thomasomys antoniobracki sp. nov. exhibits slightly long fur, 12.0 to $14.0 \mathrm{~mm}$ on the rump, and not dense; the pelage is soft with a silky texture. The hairs of the dorsal pelage are slate at the base (color 82: blackish neutral gray) and the tips ochraceous-tawny (color 26: clay color, and co- lor 33: cinnamon brown), and a band dark brown (color 219: sepia color) on the midline, the sides are somewhat more light brown. The dorsum and sides are moderately contrasting with the venter. The ventral pelage is slate at the base (color 83 dark neutral grey) and tips yellowish (color 223D: tawny olive, and color 124: buff). The ears are slightly large (26-30 mm) with a fine dark brown fur (color 219: sepia). The genal, superciliary, submental, interramal, and mystacial vibrissae are present; mystacial vibrissae long extending posteriorly well behind the pinnae when laid back against the head. Fine dark brownish hairs (patch) cover the dorsal surface of the manus and pes. Long, abundant, and whitish ungual tufts cover the claws. The hairs on the feet are blackish. The thenar is larger than the hypothenar and separated from it by a large gap. The plantar surface is squamated. Digit I of pes is the shortest; the claw reaches half the length of phalanx 1 of digit II. Digit V of pes extends to the base of claw of digit IV. The claw of digits II, III, and IV are almost the same size. The tail is monocolored (white dorsally and ventrally), longer than the body and head length. Skull. The skull of Thomasomys antoniobracki sp. nov. is comparatively large. The rostrum is long and narrow (in dorsal view) and moderately deep at the zygomatic plate level. The nasals are long, tapering backward and extending beyond the maxillary-frontal-lacrimal suture. The anterior border of the nasals is angular and projects beyond the premaxillae and the anterior border of the incisors. Rostral tube absent. The posterior margin of the premaxilla level the zygomatic notch. The bridge of the zygomatic root is high. The zygomatic notch is shallow. The zygomatic plate is broad without spine, free margins straight. The zygoma bend ventrally, but the lowest margin is unusually horizontal, placed just above the molar bridge of teeth. The zygomatic arch is divergent and slightly rounded. The jugal is long and slender. The masseteric tubercle is absent, exhibiting only a visible scar. The maxillary bridge of molars is triangular in ventral view. The lachrymal are slightly large, squarish, and slightly elevated. The interorbital region is extremely narrow, with border squarish and margins slightly diver- 
gent. These margins are not projected over the parietal except on the base of the postero-lateral process of the parietal, where almost a line is insinuated. The frontoparietal suture is semi-triangular. The petrosal bone is perforated. The interparietal is large, broad and oval, broad, exposing a small and indented occipito-parietal suture. The lambdoid crest is absent. The carotid circulation has pattern 3 (sensu Voss 1988). The alisphenoid strut is present and robust. The braincase is slightly rounded, the fronto-parietal suture is V-shaped, the squamosalparietal suture is smooth. The diastema (in lateral view) is slightly flat. The incisive foramina are narrow and long with subparallel margins. The premaxillary portion of the septum is broad and extends half the foramina length, while the maxillary portion of the septum is long and very constricted. The ethmoid foramen is small. The palatal bone is short with a small posterior process. The posterolateral palatal pits are small and placed anterior to the mesopterygoid fossa. The mesopterygoid fossa is broad, slightly expanded anteriorly. The sphenopalatine vacuities are represented as narrow slits. The parapterygoid fossa is triangular and broad with inconspicuous vacuities. The oval foramen is large. The middle lacerate foramen is open, extended anteriorly beyond the bony eustachian tube level; the alisphenoid extends from the anterior edge of tympanic bullae to the sphenopalatine foramen, and the alar fissure is shallow. The optic foramina are large and dorsal to M3. The ectotympanic bullae are elongated and moderately inflated. The ectotympanic ring is closed, although the ectotympanic margin is very narrow and weakly approaches the mastoid. The tegmen tympani overlaps the posterior suspensory process of the squamosal. The bony eustachian tube is slightly long and extend to the posterior margin of the parapterygoid fossae. The stapedial spine is long and slender and reaches the posterior margin of the alisphenoid bone. The postglenoid foramen is narrow and elongated, smaller than the subsquamosal fenestra, and both are separated by a long, straight, and slightly broad hamular process that reaches the mastoid bone to the nuchal crest. The basioccipital is sub triangular. The foramen magnum is slightly rounded. Mandible. The coronoid process is triangular-shaped and reaches the condylar process. The sigmoid notch is angular. A distinct capsular process is absent, but the lower incisor roots produce a distinct ridge and sulcus. Teeth. The upper incisors are opisthodont with yellow-orange enamel on the external surface. The molar rows are parallel. The first upper molar (M1) is large; the procingulum is anteriorly rounded and asymmetrically divided by a deep anteromedian flexus; the anteroloph is long narrow, and oriented labially and posteriorly; anteroflexus is shallow; paraflexus and metaflexus are very deep; mesoloph is slightly narrow, long, and oriented labially and posteriorly; hypoflexus is deep; posteroloph is small; the protoloph is present. The second upper molar (M2) is smaller than M1; anteroloph is long, thin, and reaches the labial surface; protoflexus is small; mesoloph is slightly short and oriented labially and posteriorly; posteroloph is very small. The third upper molar (M3) is smaller than M2; anteroloph is long, slightly broad, and reaches the labial surface; protoloph is present. The first lower molar (m1) is large; procingulum is symmetrically divided by a deep anteromedian flexid; mesolophid is narrow; hypoflexid is small and perpendicular to the labial surface; posterolophid is long, slightly broad. The second lower molar ( $\mathrm{m} 2$ ) is smaller than $\mathrm{m} 1$; anterolabial cingulum is long and narrow; mesolophid is slightly long; posterolophid is long and slightly broad. The third lower molar (m3) is smaller than $\mathrm{m} 2$; anterolabial cingulum is long and narrow.

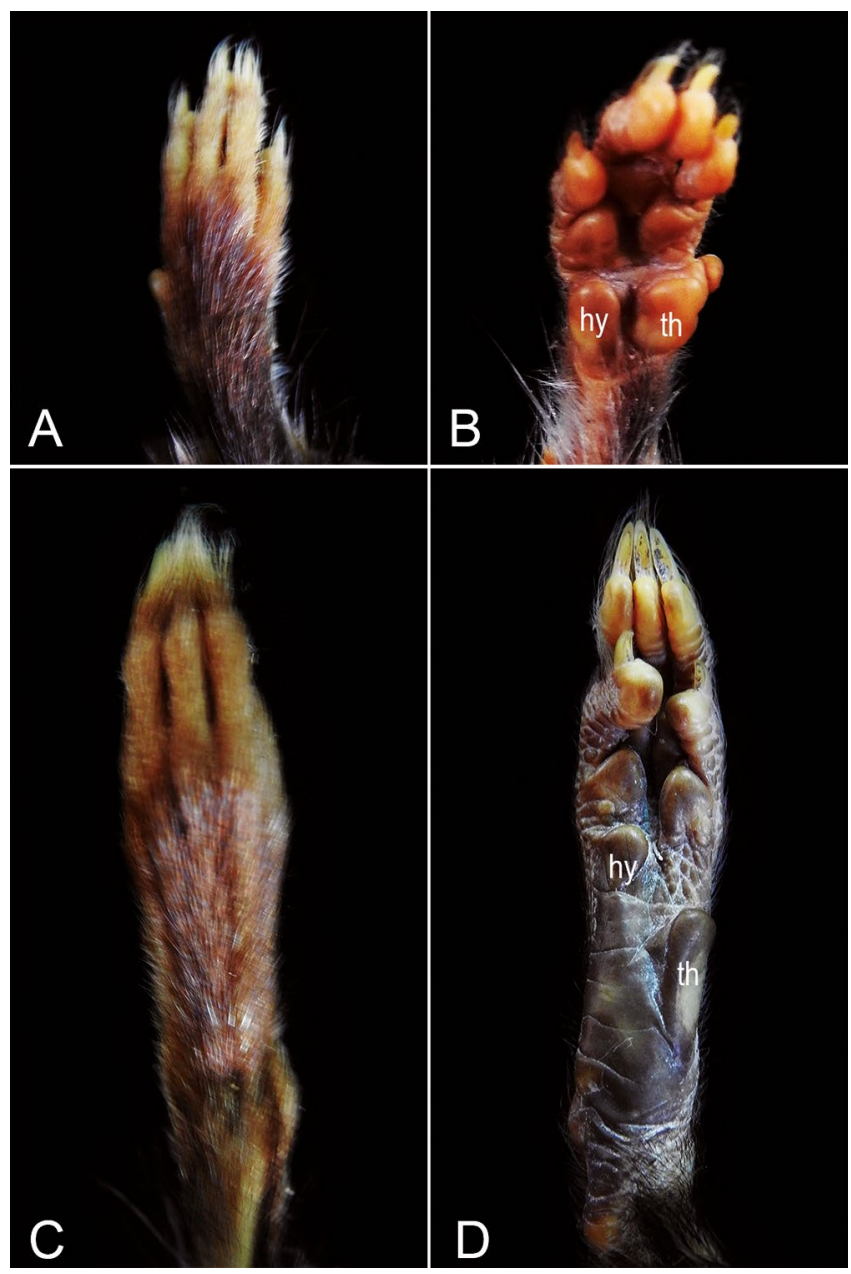

Figure 9. Dorsal and ventral views of the right manus (A, B) and right pes (C, D) of Thomasomys antoniobracki sp. nov. (MUSM 23754). Abbreviations: th, thenar; hy, hypothenar.

Etymology. Thomasomys antoniobracki sp. nov. is named in honor of Antonio José Brack Egg (Oxapampa, Pasco, June 3, 1940 - Lima, December 30, 2014), a Peruvian professor, ecologist, writer, politician, photographer, TV host, conservationist, science communicator, and researcher in biodiversity and bio-trade development. In 2006, he was appointed president of the Consejo Nacional del Ambiente (CONAM), the main environmental council up to that date in Peru. Then he was appointed as the first Minister of the new Ministry of Environment of Peru between 2008 and 2011. He stood out for his efforts to implement the new ministry and fight against illegal mining in the Peruvian Amazon. Antonio Brack was also professor at various local universities such as 
Universidad Nacional Agraria La Molina, Universidad ESAN, Universidad Ricardo Palma, Universidad Nacional de la Amazonía Peruana, Universidad Nacional Mayor de San Marcos, among others. He was the author of outstanding publications on Peruvian biodiversity and received several recognitions that include the Bárbara D'Achille National Environmental Award in 1996, Esteban Campodónico Award in 2004, and the Carlos Ponce del Prado Conservation Award in 2014.

Distribution. Thomasomys antoniobracki sp. nov. occurs in the montane forest of northern Peru, and it is currently known only from the type locality. The elevational range is from 2620 to $2720 \mathrm{~m}$.

\section{Discussion}

\section{Remarks on Thomasomys antoniobracki sp. nov.}

Our results based on morphological and molecular evidence support Thomasomys antoniobracki sp. nov. as a valid species, increasing to 47 the valid species of Thomasomys (Pacheco 2015, Brito et al. 2019, 2021). Morphologically, T. antoniobracki sp. nov. exhibits many fixed autapomorphic traits, such as the whitish tail, long and narrow stapedial spine, slightly short and triangular-shaped coronoid process, angular sigmoid notch, and protoloph present in M1. T. antoniobracki sp. nov. is also conspicuously distinct from congenerics by its large size (GLS: $45.39 \mathrm{~mm}$ ), being second only to T. apeco, currently the largest living thomasomyine rodent (GLS: $51 \mathrm{~mm}$; Leo L \& Gardner 1993). Divergence of the new species respect to another species of the "aureus" group (5.47 $\%-10.17 \%$ ) fits the threshold of interspecific variation (Baker \& Bradley 2006), similar to distances previously found among Thomasomys species using Cytb (e.g., Salazar-Bravo \& Yates 2007, Brito et al. 2019, 2021).

Thomasomys antoniobracki sp. nov. is currently considered an endemic species from the montane forest of northern Peru and known only for the type locality. However, it is likely to occur in nearby northern forests Peru and even probably in Ecuador. Although the northern montane forests seem parts of a near-continuous strip, they are presented like relict forests that house several endemic and altitudinal specialists species of flora and fauna, so they have outstanding importance for biodiversity conservation (Young \& León 1999, Weigend et al. 2013, Weigend 2013). Other Thomasomys species recorded with T. antoniobracki sp. nov. are T. caudivarius Anthony, 1923, T. taczanowskii (Thomas, 1882), and T. cf. vulcani. Following the distributional ranges presented by Pacheco (2015) for Thomasomys species, T. antoniobracki sp. nov. is possibly cohabiting with T. aureus and $T$. cinereus (Thomas, 1882).

The type locality of T. antoniobracki sp. nov. is not into a protected area, but it is at about $20 \mathrm{~km}$ (straight line) to the Santuario Nacional Tabaconas-Namballe, Cajamarca, Peru. This area is important for biodiversity conservation and protects some endangered large mammal species such as the "spectacled bear" Tremarctos ornatus and the "mountain tapir" Tapirus pinchaque (Mena \&
Velazco 2010, Cavalier et al. 2011). Although many studies have been carried out in this area (e.g., Rodríguez G 1995, Mena \& Velazco 2010, Mena \& Yagui 2019, Mena \& Pacheco 2020), the diversity of small mammals is still poorly known. Hopefully, T. antoniobracki sp. nov. is likely to occur in SN Tabaconas-Namballe. The type locality is also near the limit with Ecuador at eastern Andes (about $2.4 \mathrm{~km}$ ). In Peru, this area has not been extensively studied, and there are some mammal species from montane forests occurring near the limit that has not been yet recorded but are present in Ecuador, such as T. auricularis or T. baeops (Thomas, 1899). In contrast, Ecuador has a better knowledge of its mammal diversity in these montane forests (e.g., Aguirre Mendoza et al. 2017, Lee et al. 2018, Moreno Cárdenas \& Novillo-Gonzalez 2020), but in any of these surveys, T. antoniobracki sp. nov. has been recorded yet.

\section{Remarks on species of the "aureus" group}

The species diversity of Thomasomys is far from being known. Remarkably, the "aureus" group, as defined by Pacheco $(2003,2015)$, at the morphological level includes at least two unnamed taxa (Thomasomys sp. 1 and Thomasomys sp. 2) (Pacheco 2003). Other candidate species found with molecular information (Cytb sequences) include T. aureus "Sangay" [MN557077], T. pardignasi, and T. cf. princeps from Ecuador [MN818905]).

We found the "aureus" group is paraphyletic with members represented in two non-reciprocal clades, a clade formed by the nominal species T. aureus sensu stricto with T. antoniobracki sp. nov., T. praetor, T. aureus "Sangay," T. cf. princeps, T. auricularis, T. pardignasi, T. pyrrhonotus, and Thomasomys sp. 2; and another formed by Thomasomys sp. 1 and T. apeco, which, although weakly supported, was more related to the "notatus" and "baeops" groups. Lee et al. $(2015,2018)$ and Brito et al. $(2019,2021)$ also found a paraphyletic "aureus" group, but their representatives were very limited. Although this work is the most comprehensive phylogenetic analysis of the "aureus" group, which includes species like $T$. apeco, T. praetor, and T. pyrrhonotus, assessed for the first time in molecular analyzes, it should be considered preliminary. A complete molecular analysis of the "aureus" group would require the inclusion of T. nicefori Thomas, 1921, T. popayanus Allen, 1912, T. princeps (Thomas, 1895), T. rosalinda, and the candidate species mentioned by Pacheco (2015) and Brito et al. $(2019,2021)$.

On the other hand, it is interesting to highlight that the well-supported clade (bs: 97, pp: 1) of the "aureus" group (removing T. apeco, Thomasomys sp. 1, and Thomasomys sp. 2) include species distributed at western Río Marañón, in the Piura, Cajamarca, Lambayeque, and La Libertad departments. While T. apeco and Thomasomys sp.1 are distributed east and south of the Río Marañón. This pattern could reinforce the hypothetical biogeographic role of the Río Marañón as a barrier for sigmodontine rodents suggested by some authors (e.g., Hurtado \& Pacheco 2017; Jiménez et al. 2013; Rengifo \& Pacheco 2015; Pacheco 2015; Ruelas et al. 2021). Pacheco 2015, suggested this river and the Huancabamba Depression 
(HD) as the limit for distribution of Thomasomys species groups, such as the "incanus" (south of HD), "baeops" (north of HD), "gracilis" (south of HD), "macrotis" (between Marañón and Huallaga rivers, south of HD), and "notatus" (south of HD) groups. While "cinereus" and "aureus" groups are widely distributed across the HD and the Río Marañón. Nevertheless, a new hypothesis proposed here, based on our phylogenetic analysis, the paraphyly of the "aureus" group and T. aureus sensu lato, suggests the importance of this river on the distribution of species of this group.

Thomasomys aureus sensu lato needs to be assessed. T. aureus is the species of Thomasomys more widely distributed from central Colombia to Bolivia (Pacheco 2015, 2021). Although the type locality of the nominal form of T. aureus was not given by Tomes (1860), it was restricted to Pallatanga, Ecuador, by Pacheco (2015). Our analysis has included two specimens from the Río Tatahuazo, Bolivar province, at $30 \mathrm{~km}$ from Pallatanga (MSB 70707, 70708) cited by Pacheco (2015) as typical T. aureus. In the distribution of T. aureus, Pacheco $(2003,2015)$ included provisionally Thomasomys sp. 1 that includes populations from southeastern Peru and Bolivia and Thomasomys sp. 2 from Bolivia. Besides, there are other entities identified molecularly as related to $T$. aureus but without a morphological description. The divergence between $T$. aureus "Sangay" (KR818905: ACUNHC 1560) and T. cf. princeps (MN557077: MECN 5666) was $0.26 \pm 0.24 \%$; both collected in Parque Nacional Sangay, Ecuador (Lee et al. 2015, Brito et al. 2019) with a geographic distance between their collection locality of only $0.71 \mathrm{~km}$. Therefore, we consider both as conspecific following Pacheco (2021), who suggested that T. cf. princeps from Ecuador was not adequately identified. We tentatively call this clade T. aureus "Sangay," which is sister to T. aureus s.s. with a genetic distance of $5.64 \%$ (see Table 3). Pacheco (2021) suggested these could represent T. aureus s.s., $T$. princeps, or a new taxon, but based on our genetic data, T. aureus "Sangay" is genetically distinct from T. aureus s.s. Proper identification would require molecular comparisons with the holotype of T. princeps or topotypes from Cundinamarca. To solve the phylogenetic position of $T$. aureus s.s. and the identity of specimens recognized as T. aureus from Ecuador, we recommend obtaining the sequence of the holotype (BMNH 7.1.1.104).

Our results also give support to the recognition of $T$. auricularis as a valid species. This species has been treated as a synonym or subspecies of T. pyrrhonotus (Cabrera 1961), and following synopses (e.g., Musser \& Carleton 1993, 2005); but validated by Gyldenstolpe (1932) and later Pacheco (2003). Posteriorly, Pacheco (2015) provided detailed morphological traits that differentiate T. auricularis from T. pyrrhonotus, supported here by a genetic distance of $9.19 \%$ in our analysis.

\section{Literature cited}

Aguirre Mendoza Z, Aguirre Mendoza N, Muñoz Ch J. 2017. Biodiversidad de la provincia de Loja, Ecuador. Arnaldoa 24:523-542. https://doi.org/10.22497/arnaldoa.242.24206
Anthony HE. 1923. Preliminary report on Ecuadorean mammals No .3. American Museum Novitates 55: 1-14.

Anthony HE. 1926. Two new rodents from Bolivia. American Museum Novitates 239: 1-3.

Baker RJ and Bradley RD. 2006. Speciation in mammals and the genetic species concept. Journal of Mammalogy 87:643-662. https://doi.org/10.1644/06-MAMM-F038R2.1

Brito J, Tinoco N, Curay J, Vargas R, Reyes-Puig C, Romero V, Pardiñas UFJ. 2019. Diversidad insospechada en los Andes de ecuador: filogenia del grupo "cinereus" de Thomasomys y descripción de una nueva especie (Rodentia, Cricetidae). Mastozoología Neotropical 26:308-330. https://doi.org/10.31687/saremMN.19.26.2.0.04

Brito J, Vaca-Puente S, Koch C, Tinoco N. 2021. Discovery of the first Amazonian Thomasomys (Rodentia, Cricetidae, Sigmodontinae): a new species from the remote Cordilleras del Cóndor and Kutukú in Ecuador, Journal of Mammalogy 102:615-635. https://doi.org/10.1093/ jmammal/gyaa183

Cabrera A. 1961. Catálogo de los mamíferos de América del Sur. II (Sirenia-Perissodactyla-Artiodactyla-LagomorphaRodentia-Cetacea). Revista del Museo Argentino de Ciencias Naturales "Bernardino Rivadavia" 4:309732.

Carleton MD, Musser GG. 1989. Systematic studies of oryzomyine rodents (Muridae, Sigmodontinae): a synopsis of Microryzomys. Bulletin of the American Museum of Natural History 191:1-83.

Cavalier J, Lizcano D, Yerena E, Downer. 2011. The mountain tapir (Tapirus pinchaque) and Andean bear (Tremarctos ornatus): Two charismatic, large mammals in South American tropical montane cloud forests. In: L. Bruijnzeel, F. Scatena, and L. Hamilton, eds. Tropical montane cloud forests: Science for conservation and management. Cambridge University Press, Cambridge. Pp. 172-181.

Coues E. 1884. Thomasomys, a new subgeneric type of Hesperomys. American Naturalist 18:1275.

Darriba D, Taboada GL, Doallo R, Posada D. 2012. jModelTest 2: more models, new heuristics and parallel computing. Nature Methods 9:772. https://doi.org/10.1038/ nmeth.2109

Gyldenstolpe N. 1932. A manual of Neotropical sigmodont rodents. Almqvist \& Wiksell, 164pp.

Hurtado N, Pacheco V. 2017. Revision of Neacomys spinosus (Thomas, 1882) (Rodentia: Cricetidae) with emphasis on Peruvian populations and the description of a new species. Zootaxa 4242:401-440. https://doi. org/10.11646/zootaxa.4242.3.1

Jiménez CF, Pacheco V, Vivas D. 2013. An introduction to the systematics of Akodon orophilus Osgood, 1913 (Rodentia: Cricetidae) with the description of a new species. Zootaxa 3669:223-242. https://doi.org/10.11646/ zootaxa.3669.3.2

Kumar S, Stecher G, Tamura K. 2016. MEGA7: Molecular Evolutionary Genetics Analysis version 7.0 for bigger datasets. Molecular Biology and Evolution 33:1870-1874. https://doi.org/10.1093/molbev/msw054

Lee TE, Ritchie AR, Vaca-Puente S, Brokaw JM, Camacho MA, Burneo SF. 2015. Small mammals of Guandera Biological Reserve, Carchi Province, Ecuador and comparative Andean small mammal ecology. Occasional Papers, Museum of Texas Tech University 334:1-17. 
Lee TE, Tinoco N, Feller MJ, Gomez D, Hanson JD, Camacho AM, Burneo SF. 2018. Mammals of Yacuri National Park, Loja Province, Ecuador. Occasional Papers, Museum of Texas Tech University 357:1-17.

Leo L M, Gardner AL. 1993. A new species of a giant Thomasomys (Mammalia: Muridae: Sigmodontinae) from the Andes of northcentral Peru. Proceedings of the Biological Society of Washington 106:417-428.

Letunic I, Bork P. 2016. Interactive tree of life (iTOL) v3: an online tool for the display and annotation of phylogenetic and other trees. Nucleic Acids Research 44:W242245. https://doi.org/10.1093/nar/gkw290

Luna L, Pacheco V. 2002. A new species of Thomasomys (Muridae: Sigmodontinae) from the Andes of southeastern Peru. Journal of Mammalogy 83:834-842.

Mena JL, Pacheco V. 2020. Mountains and traits: environmental heterogeneity and mammal assemblages along an elevational gradient in the Northern Andes. Studies on Neotropical Fauna and Environment 0:1-13. https://doi.org/10.1080/01650521.2020.1851345

Mena JL, Velazco S. 2010. Comentarios sobre los mamíferos de Tabaconas. In: J.L. Mena and G. Valdivia Gozalo, eds. Conociendo el santuario nacional Tabaconas Namballe, 1st edition. World Wildlife Fund, Lima. Pp. 51-64

Mena JL, Yagui H. 2019. Coexistence and habitat use of the South American coati and the mountain coati along an elevational gradient. Mammalian Biology 98:119127. https://doi.org/10.1016/j.mambio.2019.09.004

Moreno Cárdenas PA, Novillo-Gonzalez M. 2020. First record of Thomasomys cinereus Thomas, 1882 (Rodentia, Cricetidae, Sigmodontinae) in Ecuador. Therya 11:41-45. https://doi.org/10.12933/therya-20-640

Musser G, Carleton M. 1993. Family Muridae. In: D.E. Wilson and D. Reeder, eds. Mammals species of the world: A taxonomic and geographic reference. Smithsonian Institution Press, Washington and London. Pp. 501706.

Musser GG, Carleton MD. 2005. Superfamily Muroidea. In: D.E. Wilson and D.M. Reeder (eds) Mammal Species of the World: A Taxonomic and Geographic Reference, 3rd edition. The Johns Hopkins University Press, Baltimore. Pp. 1-2142.

Nguyen L-T, Schmidt HA, von Haeseler A, Minh BQ. 2015. IQTREE: A fast and effective stochastic algorithm for estimating maximum-likelihood phylogenies. Molecular Biology and Evolution 32:268-274. https://doi. org $/ 10.1093 / \mathrm{molbev} / \mathrm{msu} 300$

Pacheco V. 2003. Phylogenetic analyses of the Thomasomyini (Muroidea: Sigmodontinae) based on morphological data. Ph.D. dissertation, The City University of New York, New York

Pacheco V. 2015. Genus Thomasomys Coues, 1884. In: J.L. Patton, U.F.J. Pardiñas, and G. D’Elía, eds. Mammals of South America. Vol. 2, Rodents. The University of Chicago Press, Chicago. Pp. 617-682

Pacheco V. 2021. Range extension of Thomasomys princeps (Thomas, 1895) (Rodentia, Sigmodontinae) and first record in Venezuela. Check List 17:385-393. https:// doi.org/10.15560/17.2.385

Rambaut A, Drummond AJ, Xie D, Baele G, Suchard MA 2018 Posterior Summarization in Bayesian Phylogenetics Using Tracer 1.7. Systematic Biology 67: 901-904. https://doi.org/10.1093/sysbio/syy032
Reig OA. 1977. A proposed unified nomenclature for the enamelled components of the molar teeth of the Cricetidae (Rodentia). Journal of Zoology 181:227-241. https:// doi.org/10.1111/j.1469-7998.1977.tb03238.x

Rengifo EM, Pacheco V. 2015. Taxonomic revision of the Andean leaf-eared mouse, Phyllotis andium Thomas 1912 (Rodentia: Cricetidae), with the description of a new species. Zootaxa 4018:349-380. https://doi. org/10.11646/zootaxa.4018.3.2

Robinson D, Hayes A, Couch S. 2021. broom: Convert Statistical Objects into Tidy Tibbles. https://CRAN.R-project. org/package=broom

Rodríguez G JJ. 1995. Mamíferos de los alrededores del Santuario Tabaconas-Namballe, Cajamarca. lista preliminar. Biotempo 2:139-140.

Ronquist F, van der Mark P, Huelsenbeck JP. 2009. Bayesian phylogenetic analysis using MRBAYES. In: P. Lemey, M. Salemi, and A.-M. Vandamme, eds. The Phylogenetic Handbook, 2nd edition. Cambridge University Press, Cambridge. Pp. 210-266.

Ronquist F, Teslenko M, van der Mark P, et al. 2012 MrBayes 3.2: Efficient Bayesian phylogenetic inference and model choice across a large model space. Systematic Biology 61:539-542. https://doi.org/10.1093/sysbio/sys029

Ruelas D, Pacheco V, Inche B, Tinoco N. 2021. A preliminary review of Nephelomys albigularis (Tomes, 1860) (Rodentia: Cricetidae), with the description of a new species from the Peruvian montane forests. Zootaxa 5027:175210. https://doi.org/10.11646/zootaxa.5027.2.3

RStudio Team. 2020. RStudio: Integrated Development for R. http://www.rstudio.com/.

Salazar-Bravo J, Yates TL 2007 A new species of Thomasomys (Cricetidae: Sigmodontinae) from central Bolivia. In: D. Kelt, E.P. Lessa, J. Salazar-Bravo, and J.L. Patton, eds. The quintessential naturalist Honoring the life and legacy of Oliver P. Pearson. University of California Press, California. Pp. 747-774.

Smith MF, Patton JL. 1993. The diversification of South American murid rodents: evidence from mitochondrial DNA sequence data for the akodontine tribe. Biological Journal of the Linnean Society 50:149-177. https:// doi.org/10.1111/j.1095-8312.1993.tb00924.x

Smithe FB. 1975. Naturalist's color guide. American Museum of Natural History, New York.

Thomas 0. 1882. On a collection of rodents from north Peru. Proceedings of The Zoological Society of London 1882 (part I): 98-111.

Thomas 0. 1886. Note on Hesperomys pyrrhorhinus, Pr. Max. Annals and Magazine of Natural History, ser. 5, 18: 421-423.

Thomas 0. 1895. On small mammals from Nicaragua and Bogota. Annals and Magazine of Natural History, ser. 6, 16:55-60.

Thomas 0. 1899. On new small mammals from South America. Annals and Magazine of Natural History, ser. 7, 3:152-155.

Thomas 0. 1900. Descriptions of two new murines from Peru and a new hare from Venezuela. Annals and Magazine of Natural History, ser. 7, 5:354-357.

Thomas 0. 1921. New Cryptotis, Thomasomys, and Oryzomys from Colombia. Annals and Magazine of Natural History, ser. 9, 8:354-357. 
Thomas O, St. Leger J. 1926. The Spedan Lewis South American Exploration .- V. Mammals obtained by Señor E. Budin in Neuquen. Annals and Magazine of Natural History, ser. 9, 18:635-641.

Tomes RF. 1860. Notes on a second collection of Mammalia made by Mr. Fraser in the Republic of Ecuador. Proceedings of The Zoological Society of London 1860:211221.

Voss RS. 1988. Systematics and ecology of ichthyomyine rodents (Muroidea): patterns of morphological evolution in a small adaptive radiation. Bulletin of the American Museum of Natural History 188:259-493.

Voss RS. 2003. A new species of Thomasomys (Rodentia: Muridae) from eastern Ecuador, with remarks on mammalian diversity and biogeography in the Cordillera Oriental. American Museum Novitates 3421:1-47.

Weigend M. 2013. Observaciones adicionales sobre la biogeografía de la zona de Amotape-Huancabamba en el norte del Perú: definiendo el límite suroriental. Revista Peruana de Biología 11:127-134. https://doi. org/10.15381/rpb.v11i2.2447

\section{Agradecimientos / Acknowledgments:}

We thank Joseph Cook and Jonathan L. Dunnum (Museum of Southwestern Biology, New Mexico) for kindly sharing with us sequences and photos of $T$. aureus sensu stricto, and to Rob Voss (American Museum of New York, New York) for allowing VP access to specimens of Thomasomys during his stay as doctoral student. We thank Omar Ruelas for preparing the ratio diagram, Richard Cadenillas, Carlos Tello, and María Peralta for collecting the specimens. This study was partially funded by the contract $N^{\circ}$ 402-PNICP-PIBA-2014 (INNOVATE-PERU) and the Universidad Nacional Mayor de San Marcos (RR 03202-R-18 and RR 03556$\mathrm{R}-19)$. This study was made under the objectives of the DIMAPA research group of the Vicerrectorado de Investigación y Posgrado of the UNMSM.

Conflicto de intereses / Competing interests:

The authors declare no conflict of interest.
Weigend M, Rodríguez EF, Arana C. 2013. Los bosques relictos del noroeste de Perú y del suroreste de Ecuador. Revista Peruana de Biología 12:185-194. https://doi. org/10.15381/rpb.v12i2.2390

Wickham H. 2019. tidyverse: Easily Install and Load the 'Tidyverse'. https://CRAN.R-project.org/package=tidyverse

Wickham H, François R, Henry L, Müller K. 2021. dplyr: A Grammar of Data Manipulation. https://CRAN.R-project. org $/$ package $=$ dplyr

Wickham H, Hester J. 2020. readr: Read Rectangular Text Data. https://CRAN.R-project.org/package=readr

Wickham H, Seidel D. 2020. scales: Scale Functions for Visualization. https://CRAN.R-project.org/package=scales

Young KR, León B. 1999. Peru's humid Eastern montane forests: an overview of their physical settings, biological diversity, human use and settlement, and conservation needs. Centre for Research on Cultural and Biological Diversity of Andean Rainforests (DIVA), Denmark, 97pp.
Rol de los autores / Authors Roles:

DR designed the research, prepared tables and figures, analyzed the data, and drafted the article. VP designed the research, analyzed the data, drafted the article, and critically reviewed the draft. All authors approved the final draft.

\section{Fuentes de financiamiento / Funding:}

INNOVATE-PERU partially funded this study under the contract $N^{\circ}$ 402-PNICP-PIBA-2014 and the Universidad Nacional Mayor de San Marcos (RR 03202-R-18 and RR 03556-R-19).The authors declare, this work not received specific funding.

\section{Aspectos éticos / legales; Ethics / legals:}

There are no ethical or legal aspects to declare when dealing with this article.

Appendix 1. List of specimens and localities used in this study. Abbreviations: AMNH, American Museum of Natural History, New York; MSB, Museum of Southwestern Biology, New Mexico; MUSM, Museo de Historia Natural, Universidad Nacional Mayor de San Marcos, Lima.

Thomasomys antoniobracki sp. nov. $(n=4)$ : PERU: Piura, Huancabamba, Campamento Alto Parramata, $4.90^{\circ} \mathrm{S}$ 79.37 W (MUSM 23754-23757).

Thomasomys aureus ( $\mathrm{n}=12$ ): ECUADOR: Bolivar: Rio Tatahuazo, $2.5 \mathrm{~km} \mathrm{E}$. of Cruz de Lizo, $1.717^{\circ} \mathrm{S} 78.983^{\circ} \mathrm{W}$ (MSB 70707); Pichincha: Guapulo, Guapulo, $0.2^{\circ} \mathrm{S} 78.483^{\circ} \mathrm{W}$ (AMNH 64701, 46577); Mt. Mojanda (south slope) $0.133^{\circ} \mathrm{N}$ $78.283^{\circ} \mathrm{W}$ (AMNH 46654); Mt. Mojanda (south slope), Piganta, $0.15^{\circ} \mathrm{N} 78.383^{\circ} \mathrm{W}$ (AMNH 46657); Mt. Mojanda (south) San José, $0.133^{\circ} \mathrm{N} 78.283^{\circ} \mathrm{W}$ (AMNH 46653); Mt. Pichincha, San Ignacio, $0.133^{\circ} \mathrm{N} 78.283^{\circ} \mathrm{W}$ (AMNH 64698); outside Quito, Charipuraz, $0.183^{\circ} \mathrm{S} 78.483^{\circ} \mathrm{W}$ (AMNH 46590, 46660); Pichincha, $0.167^{\circ} \mathrm{S} 78.6^{\circ} \mathrm{W}$ (AMNH 36280); Pichincha, above Quito, $0.167^{\circ} \mathrm{S} 78.6^{\circ} \mathrm{W}$ (AMNH 46583); Zambiza, NE Quito, $0.15^{\circ} \mathrm{S} 78.433^{\circ} \mathrm{W}$ (AMNH 46603).

Thomasomys apeco $(n=3)$ : PERU: San Martín: Mariscal Cáceres, P.N. Río Abiseo, Pampa de Cuy, $7.65^{\circ} \mathrm{S} 77.50^{\circ} \mathrm{W}$ (MUSM 7198, 7201, 7204).

Thomasomys praetor $(n=8)$ : PERU: Ancash: Pallasca, Quebrada Challhuacocha Baja, $8.25^{\circ} \mathrm{S} 77.79^{\circ} \mathrm{W}$ (MUSM 40080); Pallasca, Laguna Llamacocha, $8.25^{\circ} \mathrm{S} 77.82^{\circ} \mathrm{W}$ (MUSM 23248, 23328); Pallasca, Quebrada Chalhuacocha, Acrana, $8.24^{\circ} \mathrm{S} 77.77^{\circ} \mathrm{W}$ (MUSM 23332); Cajamarca: Cajamarca, Namococha, $6.91^{\circ} \mathrm{S} 78.42^{\circ} \mathrm{W}$ (MUSM 38354); Cajamarca, San Juan de Yerbabuena, $6.98^{\circ} \mathrm{S} 78.38^{\circ} \mathrm{W}$ (MUSM 38355); Celendín, Coñicorgue, $6.83^{\circ} \mathrm{S} 78.31^{\circ} \mathrm{W}$ (MUSM 38353); La Libertad: Sánchez Carrión, Sanagorán, $7.79^{\circ} \mathrm{S} 78.14^{\circ} \mathrm{W}$ (MUSM 46760).

Thomasomys pyrrhonotus ( $\mathrm{n}=4$ ): PERU: Cajamarca: Contumazá, Bosque Cachil, $7.39^{\circ} \mathrm{S} 78.78^{\circ} \mathrm{W}$ (MUSM 52407, 52408, 52412); Chota, San Lorenzo, $6.37^{\circ} \mathrm{S} 79.13^{\circ} \mathrm{W}$ (MUSM 39728).

Thomasomys sp. 1 ( $n=8$ ): PERU: Cusco: La Convención, Cordillera del Vilcabamba, $11.66^{\circ} \mathrm{S} 73.67^{\circ} \mathrm{W}$ (MUSM 13062); Paucartambo, Buenos Aires, carretera Paucartambo-Pillcopata, km 132, 13.18 ${ }^{\circ} \mathrm{S} 71.57^{\circ} \mathrm{W}$ (MUSM 9346); Paucartambo, Paucartambo, Challabamba, Puesto de vigilancia Acjanaco, $13.20^{\circ} \mathrm{S} 71.62^{\circ} \mathrm{W}$ (MUSM 6726, 9353, 9358, 9361, 9362); Paucartambo, Suecia, km 138.5 Carretera Shintuya, $13.10^{\circ} \mathrm{S} 71.56^{\circ} \mathrm{W}$ (MUSM 17014). 
Appendix 2. Specimens included in the phylogenetic analysis. For each terminal species, GenBank accession and catalog numbers, country, and collection locality are provided. GenBank codes in bold were gathered in this study. Museum abbreviations: MUSM, Museo de Historia Natural de la Universidad Nacional Mayor de San Marcos, Lima; FMNH, Field Museum of Natural History, Chicago; MVZ, Museum of comparative Zoology, Berkeley; QCAZ, Museo de Zoología de la Pontificia Universidad Católica del Ecuador, Quito; MEPN, Museo de Historia Natural "Gustavo Orcés V". de la Escuela Politécnica Nacional, Quito; MECN, Museo Ecuatoriano de Ciencias Naturales, Quito; UMMZ, University of Michigan Museum of Zoology, Michigan; USNM, Smithsonian National Museum of Natural History, Washington; AMNH, American Museum of Natural History, New York; UFMG, Departamento de Zoologia, Universidade Federal de Minas Gerais; MSB, Museum of Southwestern Biology, New Mexico; TTU, Museum of Texas Tech University, Lubbock. Collector abbreviations: JLP, James L. Patton; MNFS, Maria Nazareth Ferreira da Silva; TEL, Tom E. Lee, UP: Ulyses Pardiñas.

\begin{tabular}{|c|c|c|c|c|}
\hline $\begin{array}{l}\text { Genbank } \\
\text { accession }\end{array}$ & Species & $\begin{array}{l}\text { Collection/ Col- } \\
\text { lector number }\end{array}$ & Country & Locality \\
\hline AF297892 & Abrothrix olivaceus & FMNH 129876 & Chile & Aysen, General Carrera, Balmaceda, 8 km W, Fundo Esmerelda \\
\hline M35691 & Akodon boliviensis & MVZ 171607 & Peru & Puno, 12 km S Santa Rosa, 3950 m \\
\hline AF159284 & Andinomys edax & AMNH 262771 & Bolivia & $\begin{array}{l}\text { Tarija, Oconnor, } 61 \text { kilometers by road east of Tarija, Rancho } \\
\text { Tambo }\end{array}$ \\
\hline AF108690 & Auliscomys micropus & MVZ 182661 & Argentina & Rio Negro, Pampa Quemada, 12 km W Bariloche \\
\hline AF108679 & Chilomys instans & JLP 16693 & Colombia & Risaralda, Pereira, Reserva Ucumari \\
\hline AF108698 & Microryzomys minutus & MVZ 173975 & Peru & Cusco, $3 \mathrm{~km}$ E Amaybamba \\
\hline U58382 & Oecomys bicolor & MNFS 1499 & Brazil & Acre, Sobral \\
\hline AY275126 & Oxymycterus rufus & UP AC004 & Argentina & \\
\hline AF484213 & Phyllotis magister & LCM 1817 & Chile & Antofagasta, El Loa, Ojo Opache, 2 km W Calama \\
\hline KX423691 & Rhagomys longilingua & CBF 7620 & Bolivia & La Paz, Bajo Hornuni \\
\hline KY366338 & Rhipidomys latimanus & QCAZ 4784 & Ecuador & Cotopaxi, Sigchos, San Francisco de las Pampas \\
\hline KY366335 & Rhipidomys mastacalis & RM 117 & Brazil & - \\
\hline KX866980 & Sigmodon hispidus & TTU-M 80670 & USA & Texas, Morris CO, White Oak Creek WbMA (WOCWMA-2) \\
\hline DQ914643 & Thomasomys andersoni & MSB 146437 & Bolivia & Cochabamba, Corani \\
\hline DQ914644 & Thomasomys andersoni & AMNH 268734 & Bolivia & Cochabamba, Capinota, Corani \\
\hline MW788412 & Thomasomys antoniobracki sp. nov. & MUSM 23756 & Peru & Piura, Huancabamba, Minera Majaz, Campamento Alto Parramata \\
\hline MW788413 & Thomasomys antoniobracki sp. nov. & MUSM 23757 & Peru & Piura, Huancabamba, Minera Majaz, Campamento Alto Parramata \\
\hline MW788414 & Thomasomys apeco & MUSM 7199 & Peru & San Martín, Mariscal Cáceres, P.N. Río Abiseo, Los Chochos \\
\hline MW788415 & Thomasomys apeco & MUSM 7200 & Peru & San Martín, Mariscal Cáceres, P.N. Río Abiseo, Puerta de Monte \\
\hline MW788416 & Thomasomys apeco & MUSM 7201 & Peru & San Martín, Mariscal Cáceres, P.N. Río Abiseo, Pampa de Cuy \\
\hline MW788417 & Thomasomys aureus & MSB 70707 & Ecuador & Bolivar, Rio Tatahuazo, 2.5 km E of Cruz de Lizo. \\
\hline MW788418 & Thomasomys aureus & MSB 70708 & Ecuador & Bolivar, Rio Tatahuazo, 4 km E of Cruz de Lizo. \\
\hline MN557077 & Thomasomys aureus "Sangay" & $\begin{array}{l}\text { MECN 5666: } \\
\text { QCAZ } 17498\end{array}$ & Ecuador & $\begin{array}{l}\text { Chimborazo, Guamote, Cebadas, Parque Nacional Sangay, Lagu- } \\
\text { nas de Atillo [Laguna Negra] }\end{array}$ \\
\hline MN557083 & Thomasomys pardignasi & $\begin{array}{l}\text { QCAZ17489/ } \\
\text { MECN5852 }\end{array}$ & Ecuador & $\begin{array}{l}\text { Morona Santiago, San Francisco de Chinimbimi, Cordillera de } \\
\text { Kutukú } 2\end{array}$ \\
\hline MN557084 & Thomasomys pardignasi & $\begin{array}{l}\text { QCAZ17490/ } \\
\text { MECN5853 }\end{array}$ & Ecuador & $\begin{array}{l}\text { Morona Santiago, San Francisco de Chinimbimi, Cordillera de } \\
\text { Kutukú } 2\end{array}$ \\
\hline MN557061 & Thomasomys auricularis & MECN 4695 & Ecuador & Loja, Saraguro, Cerro de Arcos \\
\hline MN557124 & Thomasomys auricularis & QCAZ 6011 & Ecuador & Azuay, Cuenca, Parque Nacional El Cajas, Laguna La Toreadora \\
\hline MN557125 & Thomasomys auricularis & QCAZ 6015 & Ecuador & Azuay, Cuenca, Parque Nacional El Cajas, Laguna La Toreadora \\
\hline DQ914645 & Thomasomys australis & AMNH 268736 & Bolivia & Cochabamba, Capinota, Corani \\
\hline DQ914650 & Thomasomys australis & MSB 70447 & Bolivia & Cochabamba, Corani \\
\hline KR818877 & Thomasomys baeops & TEL 2699 & Ecuador & Carchi, San Pedro de Huaca, Páramo de la Estación Guandera \\
\hline KR818878 & Thomasomys baeops & TEL 2791 & Ecuador & Carchi, San Pedro de Huaca, Páramo de la Estación Guandera \\
\hline MN557062 & Thomasomys bombycinus & MECN 4957 & Ecuador & Carchi, Reserva Drácula \\
\hline MN557060 & Thomasomys caudivarius & $\begin{array}{l}\text { MECN } 4692- \\
\text { QCAZ } 17506\end{array}$ & Ecuador & Loja, Saraguro, Cerro de Arcos \\
\hline MN557126 & Thomasomys caudivarius & $\begin{array}{l}\text { MECN 4699- } \\
\text { QCAZ } 17508\end{array}$ & Ecuador & Loja, Saraguro, Cerro de Arcos \\
\hline MZ419564 & Thomasomys cinereus & MUSM 40973 & Peru & Cajamarca, Chota, Pagaibamba \\
\hline MZ419565 & Thomasomys cinereus & MUSM 41009 & Peru & Cajamarca, Chota, Paja Blanca \\
\hline
\end{tabular}




\begin{tabular}{|c|c|c|c|c|}
\hline KR818895 & Thomasomys cinnameus & TEL 2806 & Ecuador & Carchi, San Pedro de Huaca, Páramo de la Estación Guandera \\
\hline KR818896 & Thomasomys cinnameus & TEL 2811 & Ecuador & Carchi, San Pedro de Huaca, Páramo de la Estación Guandera \\
\hline AF108673 & Thomasomys daphne & MVZ 171502 & Peru & Puno, Agualani, 9 km N Limbani \\
\hline KY754167 & Thomasomys daphne & MVZ 171501 & Peru & Puno, Agualani, 9 km N Limbani \\
\hline EU579476 & Thomasomys erro & QCAZ 7649 & Ecuador & Napo, Archidona, Archidona, Sierra Azul, Cloud Forest Reserve \\
\hline KR818901 & Thomasomys fumeus & QCAZ 8913 & Ecuador & $\begin{array}{l}\text { Napo, Parque Nacional Sumaco Napo-Galeras, Volcán Sumaco, } \\
\text { Segundo refugio, Refugio Laguna, Entrada desde Pacto Sumaco, } \\
16 \text { km NE Wawa Sumac }\end{array}$ \\
\hline AF108674 & Thomasomys gracilis & MVZ 166668 & Peru & Cusco, 90 km SE Quillabamba \\
\hline MN557078 & Thomasomys hudsoni & MECN 5667 & Ecuador & $\begin{array}{l}\text { Chimborazo, Guamote, Parque Nacional Sangay - Bosque de } \\
\text { Polilepys }\end{array}$ \\
\hline MN557079 & Thomasomys hudsoni & MECN 5668 & Ecuador & $\begin{array}{l}\text { Chimborazo, Guamote, Parque Nacional Sangay - Bosque de } \\
\text { Polilepys }\end{array}$ \\
\hline AF108678 & Thomasomys kalinowskii & MVZ 172598 & Peru & Junín, 16 km NNE Palca \\
\hline MN557092 & Thomasomys kalinowskii & MVZ 172599 & Peru & Junín, 16 km NNE Palca \\
\hline DQ914647 & Thomasomys ladewi & MSB 68484 & Bolivia & La Paz, Rio Aceromarca \\
\hline DQ914652 & Thomasomys ladewi & MSB 68483 & Bolivia & La Paz, Rio Aceromarca \\
\hline KY754168 & Thomasomys macrotis & - & Peru & San Martín, Puerta del Monte, ca 30 km NE Los Alisos \\
\hline AF108676 & Thomasomys notatus & MVZ 166706 & Peru & Cusco, 72 km NE Paucartambo (by rd.) \\
\hline MN557128 & Thomasomys notatus & UMMZ 160588 & Peru & Cusco, 72 km NE Paucartambo (by rd.) \\
\hline KY754169 & Thomasomys onkiro & USNM 582124 & Peru & Junín, Satipo, Cordillera de Vilcabamba N, Camp One \\
\hline AF108677 & Thomasomys oreas & MVZ 166703 & Peru & Cusco, 32 km NE Paucartambo \\
\hline MN557091 & Thomasomys oreas & MVZ 166704 & Peru & Cusco, 32 km NE (by road) Paucartambo, km 112 \\
\hline KR818891 & Thomasomys paramorum & - & Ecuador & $\begin{array}{l}\text { Chimborazo, Guamote, Parque Nacional Sangay, Lagunas de } \\
\text { Atillo, Punto de Muestreo } 4\end{array}$ \\
\hline KR818893 & Thomasomys paramorum & - & Ecuador & $\begin{array}{l}\text { Chimborazo, Guamote, Parque Nacional Sangay, Lagunas de } \\
\text { Atillo, Punto de Muestreo } 4\end{array}$ \\
\hline MW788419 & Thomasomys praetor & MUSM 40080 & Peru & Ancash, Pallasca, Quebrada Challhuacocha Baja \\
\hline MW788420 & Thomasomys praetor & MUSM 40209 & Peru & Ancash, Pallasca, Cochaconchucos \\
\hline MW788421 & Thomasomys praetor & MUSM 40211 & Peru & Ancash, Pallasca, Quebrada Challhuacocha Baja \\
\hline KR818905 & Thomasomys cf. princeps & $\begin{array}{l}\text { ACUNHC 1560/ } \\
\text { QCAZ } 11939\end{array}$ & Ecuador & $\begin{array}{l}\text { Morona Santiago [Chimborazo], Guamote, Parque Nacional } \\
\text { Sangay, Lagunas de Atillo, Punto de Muestreo } 3\end{array}$ \\
\hline MW788422 & Thomasomys pyrrhonotus & MUSM 47232 & Peru & Lambayeque, Ferreñafe, Palomapampa \\
\hline MW788423 & Thomasomys pyrrhonotus & MUSM 47233 & Peru & Lambayeque, Ferreñafe, Palomapampa \\
\hline MW788424 & Thomasomys pyrrhonotus & MUSM 47234 & Peru & Lambayeque, Ferreñafe, Palomapampa \\
\hline KR818888 & Thomasomys salazari & QCAZ 11950 & Ecuador & $\begin{array}{l}\text { Chimborazo, Guamote, Parque Nacional Sangay, Lagunas de } \\
\text { Atillo, Punto de Muestreo } 4\end{array}$ \\
\hline KR818890 & Thomasomys salazari & QCAZ 11912 & Ecuador & $\begin{array}{l}\text { Chimborazo, Guamote, Parque Nacional Sangay, Lagunas de } \\
\text { Atillo, Punto de Muestreo } 3\end{array}$ \\
\hline MN557063 & Thomasomys silvestris & MECN 5238 & Ecuador & Pichincha, Tambillo \\
\hline MN557064 & Thomasomys silvestris & MECN 5394 & Ecuador & Pichincha, Tambillo \\
\hline KR818886 & Thomasomys taczanowskii & MVZ 181999 & Peru & $\begin{array}{l}\text { Cajamarca, Rio Zaña, } 2.5 \text { km NE Monte Seco on trail to Chorro } \\
\text { Blanco }\end{array}$ \\
\hline KR818887 & Thomasomys taczanowskii & MVZ 182003 & Peru & $\begin{array}{l}\text { Cajamarca, Rio Zaña, } 2.5 \text { km NE Monte Seco on trail to Chorro } \\
\text { Blanco }\end{array}$ \\
\hline KR818898 & Thomasomys ucucha & QCAZ 14969 & Ecuador & Carchi, San Pedro de Huaca, Páramo de la Estación Guandera \\
\hline KR818899 & Thomasomys ucucha & QCAZ 14930 & Ecuador & Carchi, San Pedro de Huaca, Páramo de la Estación Guandera \\
\hline KR818902 & Thomasomys vulcani & QCAZ 14925 & Ecuador & Carchi, San Pedro de Huaca, Páramo de la Estación Guandera \\
\hline KR818904 & Thomasomys vulcani & QCAZ 14926 & Ecuador & Carchi, San Pedro de Huaca, Páramo de la Estación Guandera \\
\hline U035402 & Thomasomys sp. 1 sensu Pacheco (2003) & MVZ 170076 & Peru & Cusco, Paucartambo, 72 km NE Paucartambo (by rd.) \\
\hline MW788425 & Thomasomys sp. 1 sensu Pacheco (2003) & MUSM 11999 & Peru & Cusco, Paucartambo, Paucartambo, Pillahuata \\
\hline MW788426 & Thomasomys sp. 1 sensu Pacheco (2003) & MUSM 12000 & Peru & Cusco, Paucartambo, Paucartambo, Pillahuata \\
\hline DQ914653 & Thomasomys sp. 2 sensu Pacheco (2003) & AMNH 260422 & Bolivia & $\begin{array}{l}\text { Cochabamba, Campero, ca. } 28 \text { kilometers by road west of Co- } \\
\text { marapa }\end{array}$ \\
\hline AF108685 & Wiedomys pyrrhorhinos & UFMG LC43 & Brazil & Bahia, Toca Velha, 7 km SE Canudos \\
\hline
\end{tabular}

